\title{
VIABILITY, INVARIANCE AND REACHABILITY FOR CONTROLLED PIECEWISE DETERMINISTIC MARKOV PROCESSES ASSOCIATED TO GENE NETWORKS
}

\author{
DAN GOREAC ${ }^{1}$
}

\begin{abstract}
We aim at characterizing viability, invariance and some reachability properties of controlled piecewise deterministic Markov processes (PDMPs). Using analytical methods from the theory of viscosity solutions, we establish criteria for viability and invariance in terms of the first order normal cone. We also investigate reachability of arbitrary open sets. The method is based on viscosity techniques and duality for some associated linearized problem. The theoretical results are applied to general On/Off systems, Cook's model for haploinsufficiency, and a stochastic model for bacteriophage $\lambda$.
\end{abstract}

Mathematics Subject Classification. 49L25, 60J25, 93E20, 92C42.

Received February 11, 2010. Revised November 22, 2010.

Published online April 13, 2011.

\section{INTRODUCTION}

Markov processes have been intensively used to describe variability features of various cellular processes. To our best knowledge, Markovian tools have first been employed in connection to molecular biology in [15]. The natural idea was to associate to each reaction network a pure jump model. Due to the large number of molecular species involved in the reactions, direct simulation of these models turns out to be very slow. To increase proficiency, hybrid models are adopted in [13]. They distinguish the discrete components from the "continuous" ones. Using partial Kramers-Moyal expansion, the authors of [13] replace the initial pure jump process with an appropriate piecewise deterministic Markov one.

One may reduce the complexity of PDMPs by restricting the model to some invariant set containing the initial data, whenever this is known. Compact invariant sets are also needed for efficiently implementing algorithms. Another important issue that can be approached using invariance are the stable points. In particular, a fixed point for which one finds arbitrarily small surrounding invariant sets is stable in the sense of Lyapunov.

We begin by characterizing $\varepsilon$-viability of controlled PDMPs via some associated control problem. A closed set of constraints $K$ is said to be viable (or $\varepsilon$-viable) with respect to some dynamic control system if, starting from $K$, one is able to find suitable controls keeping the trajectory in $K$ (or, at least in some arbitrarily small neighborhood of the set of constraints). Viability properties have been extensively studied in both deterministic and stochastic settings (for Brownian diffusions), starting from the pioneer work of Nagumo. The methods used to describe this property for deterministic or diffusion processes rely either on the Bouligand-Severi contingent

\footnotetext{
Keywords and phrases. Viscosity solutions, PDMP, gene networks.

1 Université Paris-Est, Laboratoire d'Analyse et Mathématiques Appliquées, UMR 8050, Boulevard Descartes, Cité Descartes, 77450 Champs-sur-Marne, France. Dan.Goreac@univ-mlv.fr
} 
cone $(c f .[2,3,16])$ or on viscosity solutions $[4-7,10,19]$. Using analytical tools from viscosity theory, we provide a geometrical characterization of $\varepsilon$-viability and invariance of some set of constraints $K$ with respect to the controlled piecewise deterministic Markov process. As for the Brownian diffusion case (cf. [7]), the criterion involves the normal cone to the set of constraints and is completely deterministic. Similar arguments allow to characterize the invariance of the set of constraints. We emphasize that these geometrical conditions can be rather easily checked for PDMPs associated to gene networks. In order to illustrate these theoretical assertions, two examples are considered. For general On/Off models, we show how the invariance criterion can be used in order to reduce the state space to a compact set. We also characterize points that can be chosen as candidates for stability (in the sense that one finds arbitrarily small surrounding regions that are invariant). Another biological example is a model for bacteriophage $\lambda$ (described in [17]). Although it is more complex, one can still use the invariance criterion to characterize candidates for stability. It turns out that only one such point exists in the absence of impulsive exterior control factors.

The second aim of the paper is to characterize the reachability property of arbitrary open sets with respect to the controlled piecewise deterministic Markov process. The criterion is obtained using viscosity methods. Recently, the paper [11] has provided a linear programming formulation for discounted control problems in the framework of SDEs driven by standard Brownian motion. The reachability problem can be connected to the value function of some appropriate piecewise deterministic control system. Using the idea in [11], we give a criterion involving the dual formulation of the linearized version of the initial problem. To illustrate this result, we consider Cook's model for haploinsufficiency introduced in [12]. Our criterion allows to prove that, starting from any arbitrary point, one reaches any arbitrarily given open region, with positive probability.

The paper is organized as follows: In Section 1.1 we briefly recall the construction of controlled PDMPs and state the main (standard) assumptions. Section 2 is devoted to the study of viability property (Sect. 2.1) and invariance (Sect. 2.2) with respect to the PDMP. The criteria involve the normal cone to the set of constraints and the characteristics of the process. Section 3 deals with the reachability property. We use a Krylov-type argument to provide some dual formulation of the associated control problem. In Section 4.1 we recall some rudiments on the PDMPs associated to a system of chemical reactions. We consider two biological examples: the On/Off model (Sect. 4.2) and the bacteriophage $\lambda$ (Sect. 4.3). We first study the compact invariant sets for the On/Off model. For a particular case (the so-called Cook model for haploinsufficiency), we prove that every open set can be reached with positive probability, starting from any initial point. In the case of the bacteriophage $\lambda$ (described in [17]), our invariance criterion allows to identify the stable point of the system. The Appendix provides the comparison principle and some stability results for viscosity solutions.

\subsection{Construction of controlled PDMPs and main assumptions}

We let $U$ be a compact metric space (the control space) and $\mathbb{R}^{N}$ be the state space, for some $N \geq 1$.

Piecewise deterministic control processes have been introduced by Davis [14]. Such processes are given by their local characteristics: a vector field $f: \mathbb{R}^{N} \times U \rightarrow \mathbb{R}^{N}$ that determines the motion between two consecutive jumps, a jump rate $\lambda: \mathbb{R}^{N} \times U \rightarrow \mathbb{R}_{+}$and a transition measure $Q: \mathbb{R}^{N} \times U \times \mathcal{B}\left(\mathbb{R}^{N}\right) \rightarrow \mathcal{P}\left(\mathbb{R}^{N}\right)$. Here $\mathcal{B}\left(\mathbb{R}^{N}\right)$ is the Borel $\sigma$-field on $\mathbb{R}^{N}$ and $\mathcal{P}\left(\mathbb{R}^{N}\right)$ stands for the family of probability measures on $\mathbb{R}^{N}$. For every $A \in \mathcal{B}\left(\mathbb{R}^{N}\right)$, the function $(u, x) \mapsto Q(x, u, A)$ is assumed to be measurable and, for every $(x, u) \in \mathbb{R}^{N} \times U$, $Q(x, u,\{x\})=0$.

We summarize the construction of controlled piecewise deterministic Markov processes (PDMP). Whenever $u \in \mathbb{L}^{0}\left(\mathbb{R}^{N} \times \mathbb{R}_{+} ; U\right)$ ( $u$ is a Borel measurable function) and $\left(t_{0}, x_{0}\right) \in \mathbb{R}_{+} \times \mathbb{R}^{N}$, we consider the ordinary differential equation

$$
\left\{\begin{array}{l}
\mathrm{d} \Phi_{t}^{t_{0}, x_{0}, u}=f\left(\Phi_{t}^{t_{0}, x_{0}, u}, u\left(x_{0}, t-t_{0}\right)\right) \mathrm{d} t, t \geq t_{0} \\
\Phi_{t_{0}, x_{0}, u}^{t_{0}}=x_{0}
\end{array}\right.
$$

We choose the first jump time $T_{1}$ such that the jump rate $\lambda\left(\Phi_{t}^{0, x_{0}, u}, u\left(x_{0}, t\right)\right)$ satisfies

$$
\mathbb{P}\left(T_{1} \geq t\right)=\exp \left(-\int_{0}^{t} \lambda\left(\Phi_{s}^{0, x_{0}, u}, u\left(x_{0}, s\right)\right) \mathrm{d} s\right) .
$$


The controlled piecewise deterministic Markov processes (PDMP) is defined by

$$
X_{t}^{x_{0}, u}=\Phi_{t}^{0, x_{0}, u}, \text { if } t \in\left[0, T_{1}\right) .
$$

The post-jump location $Y_{1}$ has $Q\left(\Phi_{\tau}^{0, x_{0}, u}, u\left(x_{0}, \tau\right), \cdot\right)$ as conditional distribution given $T_{1}=\tau$. Starting from $Y_{1}$ at time $T_{1}$, we select the inter-jump time $T_{2}-T_{1}$ such that

$$
\mathbb{P}\left(T_{2}-T_{1} \geq t / T_{1}, Y_{1}\right)=\exp \left(-\int_{T_{1}}^{T_{1}+t} \lambda\left(\Phi_{s}^{T_{1}, Y_{1}, u}, u\left(Y_{1}, s-T_{1}\right)\right) \mathrm{d} s\right) .
$$

We set

$$
X_{t}^{x_{0}, u}=\Phi_{t}^{T_{1}, Y_{1}, u}, \text { if } t \in\left[T_{1}, T_{2}\right) .
$$

The post-jump location $Y_{2}$ satisfies

$$
\mathbb{P}\left(Y_{2} \in A / T_{2}, T_{1}, Y_{1}\right)=Q\left(\Phi_{T_{2}}^{T_{1}, Y_{1}, u}, u\left(Y_{1}, T_{2}-T_{1}\right), A\right),
$$

for all Borel set $A \subset \mathbb{R}^{N}$. And so on.

Throughout the paper, unless stated otherwise, we assume the following:

(A1) The function $f: \mathbb{R}^{N} \times U \longrightarrow \mathbb{R}^{N}$ is uniformly continuous on $\mathbb{R}^{N} \times U$ and there exists a positive real constant $C>0$ such that

$$
|f(x, u)-f(y, u)| \leq C|x-y| \text {, and }|f(x, u)| \leq C,
$$

for all $x, y \in \mathbb{R}^{N}$ and all $u \in U$.

(A2) The function $\lambda: \mathbb{R}^{N} \times U \longrightarrow \mathbb{R}_{+}$is uniformly continuous on $\mathbb{R}^{N} \times U$ and there exists a positive real constant $C>0$ such that

$$
|\lambda(x, u)-\lambda(y, u)| \leq C|x-y|, \text { and } \lambda(x, u) \leq C,
$$

for all $x, y \in \mathbb{R}^{N}$ and all $u \in U$.

(A3) For each bounded uniformly continuous function $h \in B U C\left(\mathbb{R}^{N}\right)$, there exists a continuous function $\eta_{h}: \mathbb{R} \longrightarrow \mathbb{R}$ such that $\eta_{h}(0)=0$ and

$$
\sup _{u \in U}\left|\int_{\mathbb{R}^{N}} h(z) Q(x, u, \mathrm{~d} z)-\int_{\mathbb{R}^{N}} h(z) Q(y, u, \mathrm{~d} z)\right| \leq \eta_{h}(|x-y|) .
$$

(A4) For every $x \in \mathbb{R}^{N}$ and every decreasing sequence $\left(\Gamma_{n}\right)_{n \geq 0}$ of subsets of $\mathbb{R}^{N}$,

$$
\inf _{n \geq 0} \sup _{u \in U} Q\left(x, u, \Gamma_{n}\right)=\sup _{u \in U} Q\left(x, u, \cap_{n} \Gamma_{n}\right)
$$

Remark 1.1. We have kept (A3) as it appears in Soner [18]. However, this assumption may be somewhat weakened by imposing:

(A3') For each bounded uniformly continuous function $h \in B U C\left(\mathbb{R}^{N}\right)$, there exists a continuous function $\eta_{h}: \mathbb{R} \longrightarrow \mathbb{R}$ such that $\eta_{h}(0)=0$ and

$$
\sup _{u \in U}\left|\lambda(x, u) \int_{\mathbb{R}^{N}} h(z) Q(x, u, \mathrm{~d} z)-\lambda(y, u) \int_{\mathbb{R}^{N}} h(z) Q(y, u, \mathrm{~d} z)\right| \leq \eta_{h}(|x-y|) .
$$

It is obvious that whenever one assumes (A3) and $\lambda(\cdot)$ is bounded, the assumption (A3') holds true. Moreover, all the proofs in this paper can be obtained (with minor changes) when (A3') replaces (A3). 


\section{A GEOMETRIC CONDITION FOR VIABILITY AND INVARIANCE}

\subsection{Conditions for viability}

This subsection aims at characterizing the viability property of a nonempty, closed set $K \subset \mathbb{R}^{N}$. In analogy to the deterministic framework, this property is proved to be connected to some geometric condition involving the normal cone to $K$. The proof of the characterization relies on the viscosity solution concept. We begin the subsection by recalling the notions of viability (respectively $\varepsilon$-viability) and normal cone.

Definition 2.1. 1. A nonempty, closed set $K \subset \mathbb{R}^{N}$ is said to be viable with respect to the controlled piecewise deterministic Markov process $X$ if, for every initial point $x \in K$, there exists an admissible control process $u \in \mathbb{L}^{0}\left(\mathbb{R}^{N} \times \mathbb{R}_{+} ; U\right)$ such that $X_{t}^{x, u} \in K, \mathbb{P}$-almost surely, for all $t \geq 0$.

2. A nonempty, closed set $K \subset \mathbb{R}^{N}$ is said to be $\varepsilon$-viable with respect to the controlled piecewise deterministic process $X$ if, for every initial point $x \in K$ and every $\varepsilon>0$, there exists an admissible control process $u^{\varepsilon} \in$ $\mathbb{L}^{0}\left(\mathbb{R}^{N} \times \mathbb{R}_{+} ; U\right)$ such that

$$
\mathbb{E}\left[\int_{0}^{\infty} \mathrm{e}^{-t}\left(d_{K}\left(X_{t}^{x, u^{\varepsilon}}\right) \wedge 1\right) \mathrm{d} t\right] \leq \varepsilon
$$

Here, $d_{K}$ stands for the distance function to the closed set $K$.

Definition 2.2. Let $K \subset \mathbb{R}^{N}$ be a closed subset and let $x$ be a point of $K$. The normal cone to $K$ at $x$, denoted by $N_{K}(x)$, is defined as

$$
N_{K}(x)=\left\{p \in \mathbb{R}^{N}: \forall \varepsilon>0, \exists \eta>0 \text { such that } \forall y \in K \cap \bar{B}(x, \eta),\langle p, y-x\rangle \leq \varepsilon|y-x|\right\} .
$$

We recall that $\bar{B}(x, \eta)=\left\{y \in \mathbb{R}^{N}:|y-x| \leq \eta\right\}$.

The definition of the $\varepsilon$-viability property of a nonempty, closed set $K \subset \mathbb{R}^{N}$ can, alternatively, be given with respect to the value function

$$
v(x)=\inf _{u \in \mathbb{L}^{0}\left(\mathbb{R}^{N} \times \mathbb{R}_{+} ; U\right)} \mathbb{E}\left[\int_{0}^{\infty} \mathrm{e}^{-t}\left(d_{K}\left(X_{t}^{x, u}\right) \wedge 1\right) \mathrm{d} t\right],
$$

for all $x \in \mathbb{R}^{N}$. Indeed, with this notation, the set $K$ is $\varepsilon$-viable if and only if the restriction of $v$ to $K$ is zero. We consider the associated Hamilton-Jacobi integro-differential equation

$$
v(x)-d_{K}(x) \wedge 1+H(x, \nabla v(x), v)=0,
$$

for all $x \in \mathbb{R}^{N}$, where the Hamiltonian is given by

$$
H(x, p, \psi)=\sup _{u \in U}\left\{-\langle f(x, u), p\rangle-\lambda(x, u) \int_{\mathbb{R}^{N}}(\psi(z)-\psi(x)) Q(x, u, \mathrm{~d} z)\right\} .
$$

Under the assumptions (A1)-(A3), the function $v$ is known to satisfy ( $c f$. [18], Thm. 1.1), in the viscosity sense, equation (2.2). We are going to need a slightly more general definition for the viscosity subsolution (respectively supersolution) then the one used in [18].

Definition 2.3. A bounded, upper (lower) semicontinuous function $v$ is a viscosity subsolution (supersolution) of (2.2) if, for any test-function $\varphi \in C_{b}^{1}\left(N_{x}\right)$, on some neighborhood $N_{x}$ of $x \in \mathbb{R}^{N}$, whenever $x$ is a maximum (minimum) point of $v-\varphi$,

$$
v(x)-d_{K}(x) \wedge 1+H(x, \nabla \varphi(x), v) \leq 0(\geq 0) .
$$

A bounded, continuous function $v$ is a viscosity solution of (2.2) if it is both subsolution and supersolution. 
At this point, we introduce a technical assumption on the transition measure $Q$ which provides a comparison principle. It states that the probability for the post jump position to be arbitrarily far away from the prejump one is uniformly small. We emphasize that this assumption is made in order to give a simple proof for the comparison principle. However, it is not essential; one can, as an alternative, strengthen (A3) as in [1], Section 3 (see also [8]). Moreover, the main results of the paper hold true independently of this assumption, whenever a comparison principle for semicontinuous functions holds true.

(A5) We assume that

$$
\inf _{n \geq 1} \sup _{x \in \mathbb{R}^{N}, u \in U} Q\left(x, u, \mathbb{R}^{N} \backslash \bar{B}(x, n)\right)=0 .
$$

Remark 2.4. Assumption (A5) is obviously satisfied whenever the jumps do not depend on the pre-jump state $x$ nor on the control $u$. If $q \in \mathcal{P}\left(\mathbb{R}^{N}\right)$ is a probability measure, we let $Q$ be such that

$$
\int_{\mathbb{R}^{N}} h(z) Q(x, u, \mathrm{~d} z)=\int_{\mathbb{R}^{N}} h(x+z) q(\mathrm{~d} z)
$$

for all continuous function $h \in \mathcal{C}_{b}\left(\mathbb{R}^{N}\right)$ and all $x \in \mathbb{R}^{N}, u \in U$. Then

$$
Q\left(x, u, \mathbb{R}^{N} \backslash \bar{B}(x, n)\right)=q\left(\mathbb{R}^{N} \backslash \bar{B}(0, n)\right),
$$

for all $x \in \mathbb{R}^{N}, u \in U, n \geq 1$ and (A5) holds true. We emphasize that all the piecewise deterministic processes associated to chemical reactions (see Sect. 4.1) satisfy (A5).

Proposition 2.5 (comparison principle). Let $W$ be a bounded u.s.c. viscosity subsolution of (2.2) and let $V$ be a bounded l.s.c. viscosity supersolution of (2.2). Moreover, we assume that either $W$ or $V$ is uniformly continuous. Then

$$
W(x) \leq V(x),
$$

for all $x \in \mathbb{R}^{N}$.

The arguments for the proof are standard. For reader's convenience, we give the proof in the Appendix.

The main result of the subsection is the following characterization of the $\varepsilon$-viability property with respect to the controlled piecewise deterministic Markov process.

Theorem 2.6. Given a nonempty, closed set $K \subset \mathbb{R}^{N}$, the following properties are equivalent:

(i) $K$ is $\varepsilon$-viable.

(ii) The following assertions hold simultaneously:

(a) For every $x \in \partial K$, and every $p \in N_{K}(x)$,

$$
\inf _{u \in U}\left\{\langle f(x, u), p\rangle+\lambda(x, u) Q\left(x, u, K^{c}\right)\right\} \leq 0 .
$$

(b) For every $x \in \stackrel{\circ}{K}$,

$$
\inf _{u \in U}\left\{\lambda(x, u) Q\left(x, u, K^{c}\right)\right\} \leq 0 .
$$

Proof. We begin with proving that (ii) $\Rightarrow$ (i). We claim that the function

$$
V(x)=\left\{\begin{array}{l}
0, \text { if } x \in K \\
1, \text { otherwise }
\end{array}\right.
$$

is a viscosity supersolution for (2.2). By definition, $V$ is lower semi-continuous. Obviously, the supersolution condition holds true for all $x \in \mathbb{R}^{N} \backslash \partial K$. Let us now fix a point $x \in \partial K$. If $\varphi \in C_{b}^{1}\left(\mathcal{N}_{x}\right)$, for some $\mathcal{N}_{x} \subset \mathbb{R}^{N}$ 
neighborhood of $x$, is such that $(V-\varphi)$ admits a global minimum at $x$, then $\nabla \varphi(x) \in \mathcal{N}_{K}(x)$. Thus, the condition (ii) yields

$$
V(x)-\left(d_{K}(x) \wedge 1\right)+H(x, \nabla \varphi(x), V)=-\inf _{u \in U}\left\{\langle f(x, u), \nabla \varphi(x)\rangle+\lambda(x, u) Q\left(x, u, K^{c}\right)\right\} \geq 0 .
$$

It follows that $V$ is a bounded viscosity supersolution for (2.2). Using the Comparison Principle, we get

$$
v(x) \leq V(x)=0,
$$

for all $x \in K$ and the $\varepsilon$-viability of $K$ follows.

To prove the converse, we introduce, for every $m \in \mathbb{N}^{*}$, the value function $v_{m}$, defined by

$$
v_{m}(x)=m v(x)=\inf _{u \in \mathbb{L}^{0}\left(\mathbb{R}^{N} \times \mathbb{R}_{+} ; U\right)} \mathbb{E}\left[\int_{0}^{\infty} m \mathrm{e}^{-t}\left(d_{K}\left(X_{t}^{x, u}\right) \wedge 1\right) \mathrm{d} t\right],
$$

for all $x \in \mathbb{R}^{N}$. Then, Theorem 1.1 in [18] yields that $v_{m}$ is the unique bounded viscosity solution of

$$
v_{m}(x)-m\left(d_{K}(x) \wedge 1\right)+H\left(x, \nabla v_{m}(x), v_{m}\right)=0,
$$

where the Hamiltonian $H$ is given by (2.3).

Step 1. We claim that there exists a positive constant $c>0$ such that, for all $x \in K^{c}$ and all $m \geq 1$,

$$
v_{m}(x) \geq m c\left(d_{K}(x) \wedge 1\right)^{2} .
$$

We recall that on the set $\left\{T_{1}>t\right\}, X_{t}^{x, u}=\Phi_{t}^{0, x, u}$. Since $f$ is bounded, there exist a positive constant $c_{1}$ which is independent of $x, u$ and $t$ such that

$$
\left|\Phi_{t}^{0, x, u}-x\right| \leq c_{1} t
$$

for all $t \geq 0$. Thus, on the set $\left\{T_{1}>\frac{d_{K}(x) \wedge 1}{2 c_{1}}\right\}$ one gets

$$
d_{K}\left(X_{s}^{x, u}\right) \wedge 1 \geq \frac{d_{K}(x) \wedge 1}{2}>0
$$

for all $s \leq \frac{d_{K}(x) \wedge 1}{2 c_{1}}$. Using the Assumptions (A1)-(A2), one easily proves that

$$
\begin{aligned}
\mathbb{E}\left[\int_{0}^{\infty} m \mathrm{e}^{-t}\left(d_{K}\left(X_{t}^{x, u}\right) \wedge 1\right) \mathrm{d} t\right] & \geq m E\left[\int_{0}^{\frac{d_{K}(x) \wedge 1}{2 c_{1}}} \mathrm{e}^{-t} \frac{d_{K}(x) \wedge 1}{2} \mathrm{~d} t 1_{\left\{T_{1}>\frac{d_{K}(x) \wedge 1}{2 c_{1}}\right\}}\right] \\
& \geq C m\left(d_{K}(x) \wedge 1\right)^{2}
\end{aligned}
$$

Hence, (2.5) holds true for all $x \in K^{c}$.

Step 2. Let us fix $x \in \partial K$. We consider an arbitrary $p \in N_{K}(x)$ and introduce the test function

$$
\varphi(y)=\langle p, y-x\rangle-m^{\frac{1}{4}}|y-x|^{2},
$$

for all $y \in \mathbb{R}^{N}$. We let $x_{m} \in \bar{B}(x, 2)$ be such that

$$
v_{m}\left(x_{m}\right)-\varphi\left(x_{m}\right) \leq v_{m}(y)-\varphi(y),
$$


for all $y \in \bar{B}(x, 2)$. One notices that, for $m$ large enough, $x_{m} \in \bar{B}(x, 1)$. Indeed, this is a simple consequence of the fact that $v_{m}(x)=\varphi(x)=0$ and, thus,

$$
0 \leq v_{m}\left(x_{m}\right) \leq \varphi\left(x_{m}\right) \leq\left\langle p, x_{m}-x\right\rangle-m^{\frac{1}{4}}\left|x_{m}-x\right|^{2} .
$$

Moreover, for $m$ large enough, the inequality (2.6) holds true for all $y \in \mathbb{R}^{N}$. The inequalities (2.5) and (2.7) yield

This implies

$$
m c\left(d_{K}\left(x_{m}\right) \wedge 1\right)^{2} \leq v_{m}\left(x_{m}\right) \leq\left\langle p, x_{m}-x\right\rangle-m^{\frac{1}{4}}\left|x_{m}-x\right|^{2} .
$$

and

$$
\lim _{m \rightarrow \infty} m\left(d_{K}\left(x_{m}\right) \wedge 1\right)^{2}=0, \lim _{m \rightarrow \infty} x_{m}=x \text { and } \lim _{m \rightarrow \infty} m^{\frac{1}{4}}\left|x_{m}-x\right|^{2}=0,
$$

We claim that

$$
\lim _{m \rightarrow \infty} v_{m}\left(x_{m}\right)=0
$$

$$
\limsup _{m \rightarrow \infty} m^{\frac{1}{4}}\left|x_{m}-x\right|=0 .
$$

We assume that, on the contrary, there exists some positive real constant $\delta>0$ such that

$$
m^{\frac{1}{4}}\left|x_{m}-x\right|>\delta
$$

for $m$ sufficiently large. For every $m \geq 1$, we choose some $y_{m} \in K$ such that

$$
d_{K}\left(x_{m}\right)=\left|x_{m}-y_{m}\right|
$$

The equalities (2.8) imply that $\lim _{m \rightarrow \infty} y_{m}=x$. Together with the choice of $p \in N_{K}(x)$, the last limit yields

$$
\left\langle p, y_{m}-x\right\rangle \leq \frac{\delta}{2}\left|y_{m}-x\right|,
$$

for every $m$ large enough. To simplify the notation, we assume that (2.13) holds true for all $m \geq 1$. Using the inequalities (2.7), (2.13) and (2.11), we have

$$
\begin{aligned}
0 & \leq\left\langle p, x_{m}-x\right\rangle-m^{\frac{1}{4}}\left|x_{m}-x\right|^{2} \\
& \leq\left\langle p, y_{m}-x\right\rangle+\left\langle p, x_{m}-y_{m}\right\rangle-m^{\frac{1}{4}}\left|x_{m}-x\right|\left(\left|y_{m}-x\right|-\left|x_{m}-y_{m}\right|\right) \\
& \leq \frac{\delta}{2}\left|y_{m}-x\right|+|p| d_{K}\left(x_{m}\right)-\delta\left|y_{m}-x\right|+m^{\frac{1}{4}} d_{K}\left(x_{m}\right)\left|x_{m}-x\right| .
\end{aligned}
$$

Therefore,

$$
\begin{aligned}
\delta & <m^{\frac{1}{4}}\left|x_{m}-x\right| \leq m^{\frac{1}{4}}\left(\left|y_{m}-x\right|+d_{K}\left(x_{m}\right)\right) \\
& \leq\left(\frac{2}{\delta}|p|+1\right) m^{\frac{1}{4}} d_{K}\left(x_{m}\right)+\frac{2}{\delta} m^{\frac{1}{2}} d_{K}\left(x_{m}\right)\left|x_{m}-x\right| .
\end{aligned}
$$

We allow $m \rightarrow \infty$ in the inequality (2.14) and recall that (2.8) holds true to come to a contradiction. It follows that (2.10) must hold true.

We recall that the function $v_{m}$ is a bounded, continuous viscosity supersolution of (2.4) to get

$$
\begin{aligned}
& v_{m}\left(x_{m}\right)-m\left(d_{K}\left(x_{m}\right) \wedge 1\right) \\
& \geq \inf _{u \in U}\left\{\left\langle p, f\left(x_{m}, u\right)\right\rangle-2 m^{\frac{1}{4}}\left\langle x_{m}-x, f\left(x_{m}, u\right)\right\rangle+\lambda\left(x_{m}, u\right) \int_{\mathbb{R}^{N}}\left(v_{m}(z)-v_{m}\left(x_{m}\right)\right) Q\left(x_{m}, u, \mathrm{~d} z\right)\right\} .
\end{aligned}
$$


Assumption (A1) yields

$$
\begin{aligned}
& \langle p, f(x, u)\rangle=\left\langle p, f\left(x_{m}, u\right)\right\rangle-2 m^{\frac{1}{4}}\left\langle x_{m}-x, f\left(x_{m}, u\right)\right\rangle+\left\langle p, f(x, u)-f\left(x_{m}, u\right)\right\rangle+2 m^{\frac{1}{4}}\left\langle x_{m}-x, f\left(x_{m}, u\right)\right\rangle \\
& \leq\left\langle p, f\left(x_{m}, u\right)\right\rangle-2 m^{\frac{1}{4}}\left\langle x_{m}-x, f\left(x_{m}, u\right)\right\rangle+C|p|\left|x_{m}-x\right|+C m^{\frac{1}{4}}\left|x_{m}-x\right|
\end{aligned}
$$

for all $m \geq 1$ and all $u \in U$. Here $C$ is a generic real positive constant that is independent of $m \geq 1$ and $u \in U$ and may change from one line to another. Let us fix $m_{0} \geq 1$. Then, for all $m \geq m_{0}$ and all $u \in U$, by Assumptions (A2)-(A3), we obtain

$$
\begin{aligned}
& \lambda\left(x_{m}, u\right) \int_{\mathbb{R}^{N}} m c\left(d_{K}(z) \wedge 1\right)^{2} Q\left(x_{m}, u, \mathrm{~d} z\right)-\lambda\left(x_{m}, u\right) v_{m}\left(x_{m}\right) \\
& \geq c m_{0} \lambda(x, u) \int_{\mathbb{R}^{N}}\left(d_{K}(z) \wedge 1\right)^{2} Q\left(x_{m}, u, \mathrm{~d} z\right)-C\left|x_{m}-x\right| m_{0}-C v_{m}\left(x_{m}\right) \\
& \geq c m_{0} \lambda(x, u) \int_{\mathbb{R}^{N}}\left(d_{K}(z) \wedge 1\right)^{2} Q(x, u, \mathrm{~d} z)-m_{0} C \eta_{\left(d_{K} \wedge 1\right)^{2}}\left(\left|x_{m}-x\right|\right)-C\left|x_{m}-x\right| m_{0}-C v_{m}\left(x_{m}\right) \\
& \geq \lambda(x, u) Q\left(x, u, K^{c}\right)-C Q\left(x, u, K_{m_{0}}\right)-m_{0} C \eta_{\left(d_{K} \wedge 1\right)^{2}}\left(\left|x_{m}-x\right|\right)-C\left|x_{m}-x\right| m_{0}-C v_{m}\left(x_{m}\right),
\end{aligned}
$$

where we use the notation

$$
K_{m_{0}}=\left\{z \in K^{c}: d_{K}(z)<\frac{1}{\sqrt{m_{0} c}}\right\}
$$

Finally, using (2.15) and (2.16), we get

$$
\begin{aligned}
& \left\{\langle p, f(x, u)\rangle+\lambda(x, u) Q\left(x, u, K^{c}\right)\right\} \\
& \leq\left\langle p, f\left(x_{m}, u\right)\right\rangle-2 m^{\frac{1}{4}}\left\langle x_{m}-x, f\left(x_{m}, u\right)\right\rangle+C|p|\left|x_{m}-x\right|+C m^{\frac{1}{4}}\left|x_{m}-x\right| \\
& \quad+\lambda\left(x_{m}, u\right) \int_{\mathbb{R}^{N}}\left(v_{m}(z)-v_{m}\left(x_{m}\right)\right) Q\left(x_{m}, u, \mathrm{~d} z\right)+C \sup _{u \in U} Q\left(x, u, K_{m_{0}}\right) \\
& \quad+m_{0} C \eta_{\left(d_{K} \wedge 1\right)^{2}}\left(\left|x_{m}-x\right|\right)+C\left|x_{m}-x\right| m_{0}+C v_{m}\left(x_{m}\right),
\end{aligned}
$$

for all $m \geq m_{0}$ and all $u \in U$. We take in (2.17) the infimum over $u \in U$, then limsup as $m \rightarrow \infty$ and recall that the inequalities (2.8), (2.9), (2.10) hold true, to have

$$
\inf _{u \in U}\left\{\langle p, f(x, u)\rangle+\lambda(x, u) Q\left(x, u, K^{c}\right)\right\} \leq C \sup _{u \in U} Q\left(x, u, K_{m_{0}}\right)
$$

for all $m_{0} \geq 1$. Notice that $\left(K_{m_{0}}\right)$ is a decreasing sequence of sets such that $\cap_{m_{0} \geq 1} K_{m_{0}}=\phi$. Then, using Assumption (A4), the inequality (2.18) yields

$$
\inf _{u \in U}\left\{\langle p, f(x, u)\rangle+\lambda(x, u) Q\left(x, u, K^{c}\right)\right\} \leq 0 .
$$

Step 3. For $x \in \stackrel{\circ}{K}$, we take the test function $\varphi(y)=-|y-x|^{2}$ for all $y \in \mathbb{R}^{N}$. The same arguments as in Step 2 give

$$
\inf _{u \in U}\left\{\lambda(x, u) Q\left(x, u, K^{c}\right)\right\} \leq 0 .
$$

\subsection{Conditions for invariance}

Another problem, closely related to viability is the invariance of a nonempty, closed set $K \subset \mathbb{R}^{N}$. Whenever this property is satisfied, the controlled PDMP remains in $K$ independently on the control process and as soon 
as the initial datum $x \in K$. Suppose that the initial states of the model to which the PDMP is associated are known. Then, one may reduce the complexity by restricting the model to some invariant set containing the initial data. We begin by recalling the notion of invariance.

Definition 2.7. A nonempty, closed set $K \subset \mathbb{R}^{N}$ is said to be (strongly) invariant with respect to the piecewise deterministic Markov process $X$ if, for every initial point $x \in K$ and every admissible control process $u$, $X_{t}^{x, u} \in K, \mathbb{P}$-almost surely, for all $t \in \mathbb{R}_{+}$.

The invariance property is related to an optimal control problem for which the value function $v_{\text {inv }}$ is given by

$$
v_{\text {inv }}(x)=\sup _{u \in \mathbb{L}^{0}\left(\mathbb{R}^{N} \times \mathbb{R}_{+} ; U\right)} \mathbb{E}\left[\int_{0}^{\infty} \mathrm{e}^{-t}\left(d_{K}\left(X_{t}^{x, u}\right) \wedge 1\right) \mathrm{d} t\right],
$$

for all $x \in \mathbb{R}^{N}$. The main result of the section is

Theorem 2.8. Let $K \subset \mathbb{R}^{N}$ be a nonempty, closed subset. The following statements are equivalent:

(i) The set $K$ is invariant.

(ii) The value function $v_{\text {inv }}(x)=0$, for all $x \in K$.

(iii) For every $x \in \partial K$, every $p \in N_{K}(x)$, and every $u \in U$,

$$
\langle f(x, u), p\rangle+\lambda(x, u) Q\left(x, u, K^{c}\right) \leq 0 .
$$

Proof. We only need to prove that (ii) and (iii) are equivalent. We begin by proving that (iii) implies (ii). By Theorem 1.1 in [18], the function

$$
w=-v_{\text {inv }}
$$

is the unique bounded viscosity solution of the Hamilton-Jacobi integro-differential equation

$$
w(x)+d_{K}(x) \wedge 1+H(x, \nabla w, w)=0,
$$

where the Hamiltonian is given by (2.3). As in the proof of Theorem 2.6, one notices that the function

$$
V(x)=-1_{K^{c}}(x), \text { for all } x \in \mathbb{R}^{N}
$$

is a viscosity subsolution of (2.22). By the comparison principle, we get that

$$
v_{\text {inv }}(x) \leq 0,
$$

for all $x \in K$. The statement follows. The proof of the converse relies on the same arguments as Steps $1-3$ of Theorem 2.6.

\section{Reachability of open Sets}

Stability issues are very important for biological networks. For deterministic models, one can easily decide whether the system is stable, bistable, etc. However, the behavior is much less obvious for a piecewise deterministic approach. One should expect that the trajectories of the controlled PDMP starting from some region around the stable point converge to it. Alternatively, a point for which arbitrarily small surrounding regions are invariant (or at least viable) is a good candidate for stability. Thus, the issue of stability may be addressed via viability techniques. In the case of multiple stable points, given an arbitrary initial state, it would be interesting to know to which of these regions the trajectories of the PDMP are directed. The goal of this section is to address the problem of reachability.

As in the case of viability, the techniques we use rely on the theory of viscosity solutions for a class of Hamilton-Jacobi integro-differential equations. We are going to introduce a slight difference in our coefficients 
allowing to consider a control couple. To this purpose, we make the following notations: We let the vector field $\widetilde{f}: \mathbb{R}^{N} \times U \times \bar{B}(0,1) \longrightarrow \mathbb{R}^{N}$ be given by

$$
\widetilde{f}\left(x, u^{1}, u^{2}\right)=f\left(x+u^{2}, u^{1}\right),
$$

for all $x \in \mathbb{R}^{N}, u^{1} \in U$ and $u^{2} \in \bar{B}(0,1)$. Similarly, the function $\tilde{\lambda}: \mathbb{R}^{N} \times U \times \bar{B}(0,1) \longrightarrow \mathbb{R}_{+}$is given by

$$
\widetilde{\lambda}\left(x, u^{1}, u^{2}\right)=\lambda\left(x+u^{2}, u^{1}\right),
$$

and

$$
\widetilde{Q}\left(x, u^{1}, u^{2}, A\right)=Q\left(x+u^{2}, u^{1}, A+u^{2}\right),
$$

where $A+u^{2}=\left\{a+u^{2}: a \in A\right\}$, for all $x \in \mathbb{R}^{N}, u^{1} \in U, u^{2} \in \bar{B}(0,1)$ and all Borel set $A \subset \mathbb{R}^{N}$.

Remark 3.1. 1. It is obvious that, for every $h \in C_{b}\left(\mathbb{R}^{N}\right)$ and every $x \in \mathbb{R}^{N}, u^{1} \in U, u^{2} \in \bar{B}(0,1)$,

$$
\int_{\mathbb{R}^{N}} h(z) \widetilde{Q}\left(x, u^{1}, u^{2}, \mathrm{~d} z\right)=\int_{\mathbb{R}^{N}} h\left(z-u^{2}\right) Q\left(x+u^{2}, u^{1}, \mathrm{~d} z\right) .
$$

2. One can easily check that the assumptions (A1)-(A2) and (A5) hold true for the characteristic $(\tilde{f}, \tilde{\lambda}, \widetilde{Q})$ replacing $(f, \lambda, Q)$ and the set of control $U$ replaced by $U \times \bar{B}(0,1)$.

Throughout the section we are going to strengthen (A3) and assume:

(B) For each bounded uniformly continuous function $h \in B U C\left(\mathbb{R}^{N}\right)$, there exists a continuous function $\eta_{h}: \mathbb{R} \longrightarrow \mathbb{R}$ such that $\eta_{h}(0)=0$ and

$$
\sup _{u^{1} \in U, u^{2} \in \bar{B}(0,1)}\left|\int_{\mathbb{R}^{N}} h\left(z-u^{2}\right) Q\left(x+u^{2}, u^{1}, \mathrm{~d} z\right)-\int_{\mathbb{R}^{N}} h\left(z-u^{2}\right) Q\left(y+u^{2}, u, \mathrm{~d} z\right)\right| \leq \eta_{h}(|x-y|) .
$$

Remark 3.2. Similarly to Remark 1.1, one can alternatively assume:

(B') For each bounded uniformly continuous function $h \in B U C\left(\mathbb{R}^{N}\right)$, there exists a continuous function $\eta_{h}: \mathbb{R} \longrightarrow \mathbb{R}$ such that $\eta_{h}(0)=0$ and

$$
\begin{aligned}
\sup _{u^{1} \in U, u^{2} \in \bar{B}(0,1)}\left\{\begin{array}{l}
\lambda\left(x+u^{2}, u^{1}\right) \int_{\mathbb{R}^{N}} h\left(z-u^{2}\right) Q\left(x+u^{2}, u^{1}, \mathrm{~d} z\right) \\
-\lambda\left(y+u^{2}, u^{1}\right) \int_{\mathbb{R}^{N}} h\left(z-u^{2}\right) Q\left(y+u^{2}, u, \mathrm{~d} z\right)
\end{array}\right\} \\
\leq \eta_{h}(|x-y|) .
\end{aligned}
$$

For every $\varepsilon>0$, we denote by $\mathcal{E}^{\varepsilon}$ the class of measurable processes $u^{2}: \mathbb{R}^{N} \times \mathbb{R}_{+} \longrightarrow \bar{B}(0, \varepsilon)$. For every admissible control couple $\left(u^{1}, u^{2}\right) \in \mathbb{L}^{0}\left(\mathbb{R}^{N} \times \mathbb{R}_{+} ; U\right) \times \mathcal{E}^{\varepsilon}$, we let $X^{x, u^{1}, u^{2}}$ be the piecewise deterministic process associated with the characteristic $(\widetilde{f}, \widetilde{\lambda}, \widetilde{Q})$. Obviously, $X^{x, u^{1}, 0}$ is associated with $(f, \lambda, Q)$.

Let us consider an arbitrary nonempty, open set $\mathcal{O} \subset \mathbb{R}^{N}$.

Definition 3.3. Given an initial condition $x \in \mathcal{O}^{c}$ (or even $x \in \mathbb{R}^{N}$ ), the set $\mathcal{O}$ is reachable starting from $x$ if there exists some admissible control process $u$ such that the set

$$
\left\{X_{t}^{x, u, 0} \in \mathcal{O}, t \in[0, \infty)\right\}
$$

has positive probability. 
In connection to this property, we define, for every $\varepsilon \geq 0$, the value function

$$
v^{\varepsilon}(x)=\inf _{u^{1} \in \mathbb{L}^{0}\left(\mathbb{R}^{N} \times \mathbb{R}_{+} ; U\right), u^{2} \in \mathcal{E}^{\varepsilon}} \mathbb{E}\left[\int_{0}^{\infty}-\mathrm{e}^{-t}\left(d_{\mathcal{O}^{c}}\left(X_{t}^{x, u^{1}, u^{2}}+u_{t}^{2}\right) \wedge 1\right) \mathrm{d} t\right],
$$

for all $x \in \mathbb{R}^{N}$.

Remark 3.4. It is obvious that, whenever $v^{0}(x)=0$, the set $\mathcal{O}$ is not reachable starting from the point $x$. On the other hand, whenever $v^{0}(x)<0$, there exist a constant $\delta>0$, an admissible control process $u_{0} \in$ $\mathbb{L}^{0}\left(\mathbb{R}^{N} \times \mathbb{R}_{+} ; U\right)$ and $T>0$ such that $\mathbb{E}\left[\int_{0}^{T} \mathrm{e}^{-t}\left(d_{\mathcal{O}^{c}}\left(X_{t}^{x, u_{0}, 0}\right) \wedge 1\right) \mathrm{d} t\right]>\delta$. It follows that the set $\left\{X_{t}^{x, u_{0}, 0} \in \mathcal{O}\right.$, for some $\left.t \in[0, T]\right\}$ must have positive probability. Thus, $\mathcal{O}$ is reachable from $x$ if and only if $v^{0}(x)<0$.

Theorem 1.1 in Soner [18] yields that $v^{\varepsilon}$ is the unique bounded viscosity solution of the following HamiltonJacobi integro-differential equation:

$$
\begin{aligned}
0= & v^{\varepsilon}(x)+\sup _{\left|u^{2}\right| \leq \varepsilon}\left\{d_{\mathcal{O}^{c}}\left(x+u_{2}\right) \wedge 1+\sup _{u^{1} \in U}\left\{-\left\langle f\left(x+u^{2}, u^{1}\right), \nabla v^{\varepsilon}(x)\right\rangle\right.\right. \\
& \left.\left.-\lambda\left(x+u^{2}, u^{1}\right) \int_{\mathbb{R}^{N}}\left(v^{\varepsilon}(z)-v^{\varepsilon}(x)\right) \widetilde{Q}\left(x, u^{1}, u^{2}, \mathrm{~d} z\right)\right\}\right\},
\end{aligned}
$$

for all $x \in \mathbb{R}^{N}$. For the particular case $\varepsilon=0$, the value function $v^{0}$ is the unique bounded uniformly continuous viscosity solution of

$$
v^{0}(x)+d_{\mathcal{O}^{c}}(x) \wedge 1+H\left(x, \nabla v^{0}(x), v^{0}\right)=0,
$$

for all $x \in \mathbb{R}^{N}$, where the Hamiltonian $H$ is given by (2.3).

Remark 3.5. As a consequence of the definition of $\widetilde{Q}$, for every $\varepsilon>0$ and every $u^{2} \in \bar{B}(0, \varepsilon)$, the function $w(\cdot)=v^{\varepsilon}\left(\cdot-u^{2}\right)$ is a viscosity subsolution of $(3.5)$.

We get the following convergence theorem:

Theorem 3.6. There exists a decreasing function $\eta: \mathbb{R}_{+} \longrightarrow \mathbb{R}_{+}$that satisfies $\lim _{\varepsilon \rightarrow 0} \eta(\varepsilon)=0$ and such that

$$
\sup _{x \in \mathbb{R}^{N}}\left|v^{\varepsilon}(x)-v^{0}(x)\right| \leq \eta(\varepsilon)
$$

for all $\varepsilon>0$.

Proof. We recall that $v^{0}$ is uniformly continuous (cf. Thm. 1.1 in Soner [18]) and let

$$
\omega^{0}(r)=\sup \left\{\left|v^{0}(x)-v^{0}(y)\right|: x, y \in \mathbb{R}^{N},|x-y| \leq r\right\}
$$

for all $r>0$, be its continuity modulus. Let us fix $x \in \mathbb{R}^{N}$ and $\varepsilon>0$. We denote by $\Phi^{t_{0}, x_{0}, u^{1}, u^{2}}$ the flow associated to the vector field $\tilde{f}$. Standard estimates and the assumption (A1) yield the existence of some positive constant $C>0$ which is independent of $x$ and $\varepsilon>0$ such that

$$
\left|\Phi_{t}^{0, x, u^{1}, u^{2}}-\Phi_{t}^{0, x, u^{1}, 0}\right| \leq C \varepsilon
$$

for all $t \in[0,1]$, and all $\left(u^{1}, u^{2}\right) \in \mathbb{L}^{0}\left(\mathbb{R}^{N} \times \mathbb{R}_{+} ; U\right) \times \mathcal{E}^{\varepsilon}$. The constant $C$ is generic and may change from one line to another. We emphasize that throughout the proof, $C$ may be chosen independent of $x \in \mathbb{R}^{N}, \varepsilon>0$ 
and of $\left(u^{1}, u^{2}\right) \in \mathbb{L}^{0}\left(\mathbb{R}^{N} \times \mathbb{R}_{+} ; U\right) \times \mathcal{E}^{\varepsilon}$. Using the dynamic programming principle (Soner [18], equation (0.8)), for every admissible control process $u^{1} \in \mathbb{L}^{0}\left(\mathbb{R}^{N} \times \mathbb{R}_{+} ; U\right)$, the following inequality holds true

$$
v^{0}(x) \leq \mathbb{E}\left[\int_{0}^{T_{1} \wedge 1}-\mathrm{e}^{-t}\left(d_{\mathcal{O}^{c}}\left(X_{t}^{x, u^{1}, 0}\right) \wedge 1\right) \mathrm{d} t+\mathrm{e}^{-T_{1} \wedge 1} v^{0}\left(X_{T_{1} \wedge 1}^{x, u^{1}, 0}\right)\right] .
$$

We consider an arbitrary admissible control couple $\left(u^{1}, u^{2}\right) \in \mathbb{L}^{0}\left(\mathbb{R}^{N} \times \mathbb{R}_{+} ; U\right) \times \mathcal{E}^{\varepsilon}$. For simplicity, we introduce the following notations:

$$
\begin{aligned}
u_{t}^{i} & =u^{i}(x, t), i=1,2, \\
\lambda^{1}(t) & =\lambda\left(\Phi_{t}^{0, x, u^{1}, 0}, u_{t}^{1}\right), \Lambda^{1}(t)=\exp \left(-\int_{0}^{t} \lambda^{1}(s) \mathrm{d} s\right), \\
\lambda^{1,2}(t) & =\lambda\left(\Phi_{t}^{0, x, u^{1}, u^{2}}+u_{t}^{2}, u_{t}^{1}\right), \Lambda^{1,2}(t)=\exp \left(-\int_{0}^{t} \lambda^{1,2}(s) \mathrm{d} s\right),
\end{aligned}
$$

for all $t \geq 0$. We denote the right-hand member of the inequality (3.9) by $I$. Then, $I$ is explicitly given by

$$
\begin{aligned}
I= & \int_{0}^{1} \lambda^{1}(t) \Lambda^{1}(t) \int_{0}^{t}-\mathrm{e}^{-s}\left(d_{\mathcal{O}^{c}}\left(\Phi_{s}^{0, x, u^{1}, 0}\right) \wedge 1\right) \mathrm{d} s \mathrm{~d} t \\
& +\int_{0}^{1} \lambda^{1}(t) \Lambda^{1}(t) \mathrm{e}^{-t} \int_{\mathbb{R}^{N}} v^{0}(z) Q\left(\Phi_{t}^{0, x, u^{1}, 0}, u_{t}^{1}, \mathrm{~d} z\right) \mathrm{d} t \\
& +\Lambda^{1}(1) \int_{0}^{1}-\mathrm{e}^{-t}\left(d_{\mathcal{O}^{c}}\left(\Phi_{t}^{0, x, u^{1}, 0}\right) \wedge 1\right) \mathrm{d} t+\Lambda^{1}(1) \mathrm{e}^{-1} v^{0}\left(\Phi_{1}^{0, x, u^{1}, 0}\right) \\
= & I_{1}+I_{2}+I_{3}+I_{4} .
\end{aligned}
$$

Using the inequality (3.8) and the assumption (A2), one gets

$$
\begin{aligned}
& I_{1} \leq \int_{0}^{1} \lambda^{1,2}(t) \Lambda^{1,2}(t) \int_{0}^{t}-\mathrm{e}^{-s}\left(d_{\mathcal{O}^{c}}\left(\Phi_{s}^{x, u^{1}, u^{2}}+u_{s}^{2}\right) \wedge 1\right) \mathrm{d} s \mathrm{~d} t+C \varepsilon, \\
& I_{3} \leq \Lambda^{1,2}(1) \int_{0}^{1}-\mathrm{e}^{-t}\left(d_{\mathcal{O}^{c}}\left(\Phi_{t}^{x, u^{1}, u^{2}}\right) \wedge 1\right) \mathrm{d} t+C \varepsilon .
\end{aligned}
$$

For the term $I_{2}$, one has

$$
\begin{aligned}
I_{2} \leq & \int_{0}^{1} \lambda^{1,2}(t) \Lambda^{1,2}(t) \mathrm{e}^{-t} \int_{\mathbb{R}^{N}} v^{0}\left(z-u_{t}^{2}\right) Q\left(\Phi_{t}^{x, u^{1}, u^{2}}+u_{t}^{2}, u_{t}^{1}, \mathrm{~d} z\right) \mathrm{d} t+C\left(\varepsilon+\eta_{v^{0}}(C \varepsilon)+\omega^{0}(\varepsilon)\right) \\
\leq & \int_{0}^{1} \lambda^{1,2}(t) \Lambda^{1,2}(t) \mathrm{e}^{-t} \int_{\mathbb{R}^{N}} v^{\varepsilon}(z) \widetilde{Q}\left(\Phi_{t}^{x, u^{1}, u^{2}}, u_{t}^{1}, u_{t}^{2}, \mathrm{~d} z\right) \mathrm{d} t+C\left(\varepsilon+\eta_{v^{0}}(C \varepsilon)+\omega^{0}(\varepsilon)\right) \\
& +\left(\int_{0}^{1} \lambda^{1,2}(t) \Lambda^{1,2}(t) \mathrm{e}^{-t} \mathrm{~d} t\right) \sup _{z \in \mathbb{R}^{N}}\left|v^{0}(z)-v^{\varepsilon}(z)\right| .
\end{aligned}
$$

Finally,

$$
\begin{aligned}
I_{4} & \leq \Lambda^{1,2}(1) \mathrm{e}^{-1} v^{0}\left(\Phi_{1}^{x, u^{1}, u^{2}}\right)+C\left(\omega^{0}(C \varepsilon)+\varepsilon\right) \\
& \leq \Lambda^{1,2}(1) \mathrm{e}^{-1} v^{\varepsilon}\left(\Phi_{1}^{x, u^{1}, u^{2}}\right)+C\left(\omega^{0}(C \varepsilon)+\varepsilon\right)+\Lambda^{1,2}(1) \mathrm{e}^{-1} \sup _{z}\left(v^{0}(z)-v^{\varepsilon}(z)\right) .
\end{aligned}
$$


We substitute (3.10)-(3.13) in (3.9). We take the infimum over the family of $\left(u^{1}, u^{2}\right) \in \mathbb{L}^{0}\left(\mathbb{R}^{N} \times \mathbb{R}_{+} ; U\right) \times \mathcal{E}^{\varepsilon}$ and use the dynamic programming principle ( $c f$. Soner [18], equation (0.8)) to have

$$
\begin{aligned}
v^{0}(x) \leq & v^{\varepsilon}(x)+C\left(\varepsilon+\eta_{v^{0}}(C \varepsilon)+\omega^{0}(C \varepsilon)\right) \\
& +\left(\int_{0}^{1} \lambda^{1,2}(t) \Lambda^{1,2}(t) \mathrm{e}^{-t} \mathrm{~d} t+\Lambda^{1,2}(1) \mathrm{e}^{-1}\right) \sup _{z}\left(v^{0}(z)-v^{\varepsilon}(z)\right) .
\end{aligned}
$$

We notice that

$$
\int_{0}^{1} \lambda^{1,2}(t) \Lambda^{1,2}(t) \mathrm{e}^{-t} \mathrm{~d} t+\Lambda^{1,2}(1) \mathrm{e}^{-1}=1-\int_{0}^{1} \mathrm{e}^{-\int_{0}^{t} \widetilde{\lambda}\left(\Phi_{s}^{x, u^{1}, u^{2}}, u_{s}^{1}, u_{s}^{2}\right) \mathrm{d} s} \mathrm{e}^{-t} \mathrm{~d} t \leq 1-\mathrm{e}^{-\left(\lambda^{\max }+1\right)} .
$$

Thus,

$$
v^{0}(x)-v^{\varepsilon}(x) \leq C\left(\varepsilon+\eta_{v^{0}}(C \varepsilon)+\omega^{0}(C \varepsilon)\right)+\left(1-\mathrm{e}^{-\left(\lambda^{\max }+1\right)}\right) \sup _{z}\left(v^{0}(z)-v^{\varepsilon}(z)\right) .
$$

Here, $\lambda^{\max }=\sup \left\{\lambda(x, u): x \in \mathbb{R}^{N}, u \in U\right\}<\infty$. The conclusion follows by taking the supremum over $x \in \mathbb{R}^{N}$ and recalling that $C$ is independent of $x$ and $\varepsilon>0$.

We introduce the function $\mu^{*}: \mathbb{R}^{N} \longrightarrow \mathbb{R}$ defined by

$$
\begin{aligned}
\mu^{*}(x) & =\sup \left\{\mu \in \mathbb{R}: \exists \varphi \in C_{b}^{1}\left(\mathbb{R}^{N}\right) \text { such that } \forall(y, u) \in \mathbb{R}^{N} \times U,\right. \\
& \left.\mu \leq \mathcal{U}^{u} \varphi(y)-\left(d_{\mathcal{O}^{c}}(y) \wedge 1\right)+(\varphi(x)-\varphi(y))\right\}
\end{aligned}
$$

where

$$
\mathcal{U}^{u} \varphi(y)=\langle\nabla \varphi(y), f(y, u)\rangle+\lambda(y, u) \int_{\mathbb{R}^{N}}(\varphi(z)-\varphi(y)) Q(y, u, \mathrm{~d} z),
$$

for all $y \in \mathbb{R}^{N}$. This function is inspired by the results in [11]. It corresponds to the dual form of some linearized formulation for the discounted control problem. In fact, one can interpret the initial problem by using occupational measures. In a second step, the set of occupational measures can be enlarged to a set of measures satisfying appropriate conditions. These conditions involve the infinitesimal generator of the underlying process and can be interpreted as a classical constraint. Minimizing on this set leads to the same value function. Duality techniques then allow to give a formulation much like $\mu^{*}$ (but for generators associated to Brownian diffusion processes).

The main result of the section gives the equality between the reachability value function $v^{0}$ and $\mu^{*}$.

Theorem 3.7. For every $x \in \mathbb{R}^{N}$, the equality

$$
v^{0}(x)=\mu^{*}(x)
$$

holds true.

Proof. We begin by proving that

$$
v^{0}(x) \geq \mu^{*}(x),
$$

for all $x \in \mathbb{R}^{N}$. We fix $x \in \mathbb{R}^{N}$ and $(\mu, \varphi) \in \mathbb{R} \times C_{b}^{1}\left(\mathbb{R}^{N}\right)$ such that

$$
\mu \leq \mathcal{U}^{u} \varphi(y)-\left(d_{\mathcal{O}^{c}}(y) \wedge 1\right)+(\varphi(x)-\varphi(y)),
$$

for all $y \in \mathbb{R}^{N}, u \in U$. Then, for every $u \in \mathbb{L}^{0}\left(\mathbb{R}^{N} \times \mathbb{R}_{+} ; U\right)$,

$$
\mu \leq \mathcal{U}^{u_{t}} \varphi\left(X_{t}^{x, u, 0}\right)+\varphi(x)-\varphi\left(X_{t}^{x, u, 0}\right)-\left(d_{\mathcal{O}^{c}}\left(X_{t}^{x, u, 0}\right) \wedge 1\right),
$$


for all $t \geq 0$. By abuse of notation,

$$
\mathcal{U}^{u_{t}} \varphi\left(X_{t}^{x, u, 0}\right)=\mathcal{U}^{u\left(X_{T_{i}}^{x, u, 0}, t-T_{i}\right)} \varphi\left(X_{t}^{x, u, 0}\right), \text { whenever } T_{i} \leq t<T_{i+1},
$$

where $T_{i}$ are the jump times appearing in Section 1.1. Using Itô's formula (cf. Thm. 31.3 in [14]), the last inequality yields

$$
\begin{aligned}
\mu \leq & \lim _{T \rightarrow \infty} \mathbb{E}\left[\int_{0}^{T} \mathrm{e}^{-t}\left(\mathcal{U}^{u_{t}} \varphi\left(X_{t}^{x, u, 0}\right)-\varphi\left(X_{t}^{x, u, 0}\right)\right) \mathrm{d} t\right] \\
& +\varphi(x)+\mathbb{E}\left[\int_{0}^{\infty}-\mathrm{e}^{-t}\left(d_{\mathcal{O}^{c}}\left(X_{t}^{x, u, 0}\right) \wedge 1\right) \mathrm{d} t\right] \\
= & \lim _{T \rightarrow \infty} \mathrm{e}^{-T} \mathbb{E}\left[\varphi\left(X_{T}^{x, u, 0}\right)\right]+\mathbb{E}\left[\int_{0}^{\infty}-\mathrm{e}^{-t}\left(d_{\mathcal{O}^{c}}\left(X_{t}^{x, u, 0}\right) \wedge 1\right) \mathrm{d} t\right] \\
= & \mathbb{E}\left[\int_{0}^{\infty}-\mathrm{e}^{-t}\left(d_{\mathcal{O}^{c}}\left(X_{t}^{x, u, 0}\right) \wedge 1\right) \mathrm{d} t\right] .
\end{aligned}
$$

We recall the definition (3.14) of $\mu^{*}(x)$ and the inequality (3.17) follows from (3.18).

In order to complete the proof of the theorem, we still have to prove that

$$
\mu^{*}(x) \geq v^{0}(x)
$$

Let us consider $\left(\rho_{\varepsilon}\right)$ a sequence of standard mollifiers $\rho_{\varepsilon}(y)=\frac{1}{\varepsilon^{N}} \rho\left(\frac{y}{\varepsilon}\right), y \in \mathbb{R}^{N}, \varepsilon>0$, where $\rho \in C^{\infty}\left(\mathbb{R}^{N}\right)$ is a positive function such that

$$
\operatorname{Supp}(\rho) \subset \bar{B}(0,1) \text { and } \int_{\mathbb{R}^{N}} \rho(x) \mathrm{d} x=1
$$

We introduce the functions

$$
V^{\varepsilon}=v^{\varepsilon} * \rho_{\varepsilon},
$$

for all $\varepsilon>0$. We claim that these functions are (viscosity) subsolutions of (3.5). The proof follows the same arguments as Lemma 2.7 in Barles and Jakobsen [9]. For convenience, we give the proof in the Appendix. Using the fact that $V^{\varepsilon}$ is a subsolution of (3.5), one gets

$$
V^{\varepsilon}(x) \leq \mu^{*}(x) .
$$

It follows, from (3.6) that

$$
\left(v^{0} * \rho_{\varepsilon}\right)(x) \leq \mu^{*}(x)+\eta(\varepsilon) .
$$

We allow $\varepsilon \rightarrow 0$ in the last inequality, and recall that $v^{0}$ is continuous, to finally get (3.19). The proof of the theorem is now complete.

The previous result gives the following interesting characterization of the reachability of the set $\mathcal{O}$ :

Criterion 3.8. Let $x \in \mathbb{R}^{N}$ be an arbitrary initial state. Then the controlled piecewise deterministic Markov process starting from $x$ reaches $\mathcal{O}$ if and only if there exists $n \in \mathbb{N}^{*}$ such that for every $\varphi \in C_{b}^{1}\left(\mathbb{R}^{N}\right)$ there exist $u \in U, y \in \mathbb{R}^{N}$ such that

$$
\mathcal{U}^{u} \varphi(y)-d_{\mathcal{O}^{c}}(y) \wedge 1+(\varphi(x)-\varphi(y))<-n^{-1} .
$$




\section{Biological EXAmples}

\subsection{Biochemical reactions and mathematical assumptions}

We begin by recalling some rudiments on piecewise deterministic Markov processes associated to gene networks. For further contributions on gene networks modelling the reader is referred to [13]. We suppose that the biological evolution is given by a family of genes $\mathcal{G}=\left\{g_{i}: i=1, N\right\}$ interacting through a finite set of reactions $\mathcal{R}$. Every reaction $r \in \mathcal{R}$ can be represented as

$$
\alpha_{1}^{r} g_{1}+\alpha_{2}^{r} g_{2}+\ldots+\alpha_{N}^{r} g_{N} \stackrel{k_{r}}{\longrightarrow} \beta_{1}^{r} g_{1}+\ldots+\beta_{N}^{r} g_{N}
$$

and it specifies that $\alpha_{i}^{r}$ molecules of $i$ type (with $1 \leq i \leq N$ ) called reactants interact in order to form the products $\left(\beta_{i}^{r}\right.$ molecules of $i$ type, with $\left.1 \leq i \leq N\right)$. The reaction does not occur instantaneously and one needs to specify the reaction speed $k_{r}>0$. Also, the presence of all species is not required $\left(\alpha_{i}^{r}, \beta_{i}^{r} \in \mathbb{N}\right.$, for all $\left.1 \leq i \leq N\right)$. The species are partitioned in two classes called continuous, respectively discrete component. This partition (for further considerations, see [13]) induces a partition of the reactions. In sum, we distinguish between reactions contributing to the continuous flow $\left(\mathcal{C}=\left\{1,2, \ldots, M_{1}\right\}\right)$ and jump reactions $\left(\mathcal{J}=\left\{M_{1}+1, \ldots, \operatorname{card}(\mathcal{R})\right\}\right)$. To every reaction $r \in \mathcal{R}$, one associates:

1) a stoichiometric column vector $\theta^{r}=\beta^{r}-\alpha^{r} \in \mathbb{R}^{N}$;

2) a propensity function $\lambda_{r}: \mathbb{R}^{N} \longrightarrow \mathbb{R}_{+}$.

For a $\mathcal{C}$-type reaction, $\lambda_{r}(x)=k_{r} \prod_{i=1}^{N} x_{i}^{\alpha_{i}^{r}}$, for all $x \in \mathbb{R}^{N}$.

For a $\mathcal{J}$-type reaction, one should require further regularity as $x_{i} \rightarrow 0$. The jump mechanism will specify that the number of molecules of type $i$ diminishes by $\alpha_{i}^{r}$. Therefore, in order to insure positive components, rather then introducing $\lambda_{r}(x)$ as for continuous reactions, one could consider

$$
\lambda_{r}(x)=k_{r} \prod_{\substack{i=1 \\ \alpha_{i}^{r}>0}}^{N} x_{i}^{\alpha_{i}^{r}} \chi\left(\frac{x_{i}}{\alpha_{i}^{r}}\right),
$$

for some regular function $\chi$ such that $0 \leq \chi \leq 1, \chi(y)=0$, for $0 \leq y \leq 1$ and $\chi(y)=1$, for $y \geq 1+$ err (where err is a positive constant).

The next step consists in the construction of two matrices $M_{1}$ whose columns are the vectors $\alpha^{r}$, where $r \in \mathcal{C}$, respectively $M_{2}$ whose columns are the vectors $\alpha^{r}$, where $r \in \mathcal{J}$. The flow is the given by

$$
f(x)=M_{1} \times\left(\lambda_{1}(x), \lambda_{2}(x), \ldots, \lambda_{M_{1}}(x)\right),
$$

the jump intensity

$$
\lambda(x)=\sum_{r \in J} \lambda_{r}(x)
$$

and, whenever $\lambda(x)>0$, the transition measure $Q$ is given by

$$
Q(x, \mathrm{~d} z)=\sum_{r \in \mathcal{J}} \frac{\lambda_{r}(x)}{\lambda(x)} \delta_{x+\theta^{r}}(\mathrm{~d} z) .
$$

One can suppose that all $\lambda_{r}$ are bounded by a reasonable constant $\lambda^{\max }>0$, by replacing $\lambda_{r}(x)$ by $\lambda_{r}(x) \wedge \lambda^{\max }$. Then, it is obvious that (A1) and (A2) hold true. If $h \in B U C\left(\mathbb{R}^{N}\right)$,

$$
\begin{aligned}
& \left|\lambda(x+e) \int_{\mathbb{R}^{N}} h(z-e) Q(x+e, \mathrm{~d} z)-\lambda(y+e) \int_{\mathbb{R}^{N}} h(z-e) Q(y+e, \mathrm{~d} z)\right| \\
& \leq \sum_{r \in \mathcal{J}}\left|\lambda_{r}(x+e) h\left(x+\theta^{r}\right)-\lambda_{r}(y+e) h\left(y+\theta^{r}\right)\right| \leq c\left(|x-y|+\omega_{h}(|x-y|)\right),
\end{aligned}
$$


for all $x, y, e \in \mathbb{R}^{N}$, where $c$ depends on the Lipschitz constant of $\lambda_{r}, \lambda^{\max }$ and $\|h\|_{\infty}$ and $\omega_{h}$ is the continuity modulus of $h$. This implies that (B') (and a fortiori (A3')) hold true. The assumption (A4) is a simple consequence of the fact that $Q(x, \cdot)$ is a probability measure for every $x \in \mathbb{R}^{N}$. Also, one easily notices that

$$
Q\left(x, \mathbb{R}^{N} \backslash \bar{B}\left(x, \sup _{r \in \mathcal{J}}\left|\theta^{r}\right|\right)\right)=0
$$

which implies (A5). It follows that all the assumptions we have made throughout the paper are naturally satisfied for piecewise deterministic systems associated to regulatory gene networks.

\subsection{On/Off model}

A two-state model is often employed to describe different situations in the molecular biology. Usually, the two states describe either the presence or the absence of some rare molecular specie. Whenever the gene $\gamma$ is inactive (represented by $\gamma=0$ ), the molecule $X$ degrades at a rate $r_{0}$, while as, whenever $\gamma$ is active $(\gamma=1)$, the molecule $X$ increases at a rate proportional to some given $r_{1}$.

From the mathematical point of view, the system will be given by a process $(X(t), \gamma(t))$ on the state space $E=\mathbb{R} \times\{0 ; 1\}$. The component $X(t)$ follows a differential dynamic depending on the hidden variable

$$
\frac{\mathrm{d} X}{\mathrm{~d} t}=\left\{\begin{array}{c}
-r_{0}(X), \text { if } \gamma(t)=0 \\
r_{1}(X), \text { if } \gamma(t)=1
\end{array}\right.
$$

where $r_{0}(x) \geq 0$ is a bounded, Lipschitz-continuous consumption term and $r_{1}(x) \geq 0$ is a bounded, Lipschitz continuous production term. To be more precise, the PDMP associated to the model has the characteristic $(f, \lambda, Q)$ given by $f_{\gamma}(x)=-r_{0}(x)(1-\gamma)+r_{1}(x) \gamma, \lambda_{\gamma}(x)=\lambda_{\gamma}, Q(\gamma, x ; A)=Q((\gamma, x) ; A)=\delta_{((1-\gamma), x)}(A)$, for all $\gamma \in\{0,1\}, x \in \mathbb{R}$, and all $A \subset \mathbb{R}^{2}$. The vector field for the $\gamma$ component can be considered to be 0 . One should expect 0 -consumption whenever $X=0$ and $\gamma=0$ i.e. $r_{0}(0)=0$, and no production whenever $X=\alpha_{\max }$ (some maximum level) and $\gamma=1$, i.e. $r_{1}\left(\alpha_{\max }\right)=0$. The assumptions (A1)-(A5) are obviously satisfied.

Proposition 4.1. The set $K=\left[0, \alpha_{\max }\right] \times\{0,1\}$ is invariant with respect to the PDP associated to the On/Off model.

Proof. If $x \in\left[0, \alpha_{\max }\right]$, then, by the definition of $Q$,

$$
Q\left((0, x), K^{c}\right)=Q\left((1, x), K^{c}\right)=0 .
$$

One notices that $N_{\left[0, \alpha_{\max }\right]}(0)=\mathbb{R}_{-}$and $N_{\left[0, \alpha_{\max }\right]}\left(\alpha_{\max }\right)=\mathbb{R}_{+}$. We recall that $N_{\left[0, \alpha_{\max }\right]}(x)$ stands for the normal cone to $\left[0, \alpha_{\max }\right]$ at $x \in\left[0, \alpha_{\max }\right]$. For every $p \leq 0$,

$$
p f_{0}(0)=-p r_{0}(0)=0 \text { and } p f_{1}(0) \leq 0 .
$$

For every $p \geq 0$,

$$
p f_{0}\left(\alpha_{\max }\right)=-p r_{0}\left(\alpha_{\max }\right) \leq 0 \text { and } p f_{1}\left(\alpha_{\max }\right)=0 .
$$

Thus, by applying Theorem 2.8, one gets the invariance of $K$.

Remark 4.2. The arguments of the previous proposition yield that $[a, b] \times\{0,1\}$ is invariant if and only if

$$
r_{0}(a)=r_{1}(b)=0 .
$$

Therefore, a point $x_{0}$ is stable whenever one is able to find a sequence $\varepsilon \searrow 0$ such that $r_{0}\left(x_{0}-\varepsilon\right)=r_{1}\left(x_{0}+\varepsilon\right)=0$. In particular, a necessary condition is that $r_{0}\left(x_{0}\right)=r_{1}\left(x_{0}\right)=0$. 
We now focus on Cook's model introduced in [12] for stochastic gene expression and its implications on haploinsufficiency. This basic model of gene expression, product accumulation and product degradation can be given by the following reaction system:

$$
\mathbf{G} \underset{k_{d}}{\stackrel{k_{a}}{\rightleftarrows}} \mathbf{G}^{*} \stackrel{J_{p}}{\longrightarrow} \stackrel{\text { P }}{\longrightarrow} \stackrel{k_{p}}{\longrightarrow}
$$

This model considers a gene to switch randomly between inactive state $(\mathrm{G})$ and active state $\left(\mathrm{G}^{*}\right)$. The activation (respectively deactivation) rate is denoted by $k_{a}$ (respectively $k_{d}$ ). When active, each gene expresses a product $(\mathrm{P})$ at a rate $J_{p}$. The product is degraded at rate $k_{p}$. One can represent this model as a particular case of the On/Off system by considering

$$
r_{0}(x)=k_{p} x, r_{1}(x)=J_{p}-k_{p} x, \lambda_{0}=k_{a}, \lambda_{1}=k_{d}, \text { and } \alpha_{\max }=\frac{J_{p}}{k_{p}} .
$$

The following result is a consequence of Criterion 3.8:

Proposition 4.3. For every real constants $a, b$ such that $0<a<b<\alpha_{\max }$, we let $\mathcal{O}=(a, b) \times\{0,1\}$. Then, for every $x \in\left(0, \alpha_{\max }\right)$, the set $\mathcal{O}$ is reachable with respect to the PDMP associated with Cook's model starting from $(0, x)$.

Proof. Let us fix $x \in\left(0, \alpha_{\max }\right)$. One seeks to apply Criterion 3.8. We reason by contradiction and assume, that, for every $n \in \mathbb{N}^{*}$, there exists $\varphi_{n}, \psi_{n} \in C_{b}^{1}(\mathbb{R})$ such that

$$
\left\{\begin{array}{c}
-n^{-1} \leq-\varphi_{n}^{\prime}(y) k_{p} y+k_{a} \psi_{n}(y)-\left(1+k_{a}\right) \varphi_{n}(y)-d_{[0, a] \cup\left[b, \alpha_{\max }\right]}(y)+\varphi_{n}(x), \\
-n^{-1} \leq \psi_{n}^{\prime}(y)\left(J_{p}-k_{p} y\right)-\left(1+k_{d}\right) \psi_{n}(y)+k_{d} \varphi_{n}(y)-d_{[0, a] \cup\left[b, \alpha_{\max }\right]}(y)+\varphi_{n}(x),
\end{array}\right.
$$

for all $y \in[0,1]$. We multiply the first inequality by $y^{\frac{1+k_{a}-k_{p}}{k_{p}}}$ and integrate on $(0, z]$, for $z>0$, to get

$$
k_{p} z^{\frac{1+k_{a}}{k_{p}}} \varphi_{n}(z) \leq \frac{k_{p}}{1+k_{a}} z^{\frac{1+k_{a}}{k_{p}}}\left(n^{-1}+\varphi_{n}(x)\right)+k_{a} \int_{0}^{z} y^{\frac{1+k_{a}-k_{p}}{k_{p}}} \psi_{n}(y) \mathrm{d} y-\int_{0}^{z} y^{\frac{1+k_{a}-k_{p}}{k_{p}}} d_{[0, a] \cup\left[b, \alpha_{\max }\right]}(y) \mathrm{d} y
$$

or again

$$
\varphi_{n}(z) \leq \frac{1}{1+k_{a}}\left(n^{-1}+\varphi_{n}(x)\right)+\frac{k_{a}}{k_{p}} \frac{\int_{0}^{z} y^{\frac{1+k_{a}-k_{p}}{k_{p}}} \psi_{n}(y) \mathrm{d} y}{z^{\frac{1+k_{a}}{k_{p}}}}-\frac{1}{k_{p}} \frac{\int_{0}^{z} y^{\frac{1+k_{a}-k_{p}}{k_{p}}} d_{[0, a] \cup\left[b, \alpha_{\max }\right]}(y) \mathrm{d} y}{z^{\frac{1+k_{a}}{k_{p}}}},
$$

for all $z \in\left(0, \alpha_{\max }\right]$. We multiply the second inequality in $(4.2)$ by $\left(J_{p}-k_{p} y\right)^{\frac{1+k_{d}-k_{p}}{k_{p}}}$ and integrate on $\left[z, \alpha_{\max }\right)$, for $z<\alpha_{\max }$, to get

$$
\begin{aligned}
\psi_{n}(z) \leq & \frac{1}{1+k_{d}}\left(n^{-1}+\varphi_{n}(x)\right)+k_{d} \frac{\int_{z}^{\alpha_{\max }}\left(J_{p}-k_{p} y\right)^{\frac{1+k_{d}-k_{p}}{k_{p}}} \varphi_{n}(y) \mathrm{d} y}{\left(J_{p}-k_{p} z\right)^{\frac{1+k_{d}}{k_{p}}}} \\
& -\frac{\int_{z}^{1}\left(J_{p}-k_{p} y\right)^{\frac{1+k_{d}-k_{p}}{k_{p}}} d_{[0, a] \cup\left[b, \alpha_{\max }\right]}(y) \mathrm{d} y}{\left(J_{p}-k_{p} z\right)^{\frac{1+k_{d}}{k_{p}}}},
\end{aligned}
$$

for all $z \in\left[0, \alpha_{\max }\right)$. We denote by $a_{n}$ the maximum value of $\varphi_{n}$ on $\left[0, \alpha_{\max }\right]$. It follows that

$$
\psi_{n}(z) \leq \frac{1}{1+k_{d}}\left(n^{-1}+\varphi_{n}(x)\right)+\frac{k_{d}}{1+k_{d}} a_{n}-\frac{\int_{z}^{\alpha_{\max }}\left(J_{p}-k_{p} y\right)^{\frac{1+k_{d}-k_{p}}{k_{p}}} d_{[0, a] \cup\left[b, \alpha_{\max }\right]}(y) \mathrm{d} y}{\left(J_{p}-k_{p} z\right)^{\frac{1+k_{d}}{k_{p}}}},
$$


for all $z \in\left[0, \alpha_{\max }\right)$. We substitute the last inequality in (4.3), to have

$$
\varphi_{n}(z) \leq \frac{k_{a}+k_{d}+1}{\left(1+k_{a}\right)\left(1+k_{d}\right)}\left(n^{-1}+\varphi_{n}(x)\right)+\frac{k_{a} k_{d}}{\left(1+k_{a}\right)\left(1+k_{d}\right)} a_{n}-f(z),
$$

for all $z \in\left(0, \alpha_{\max }\right)$. The function $f$ is defined by

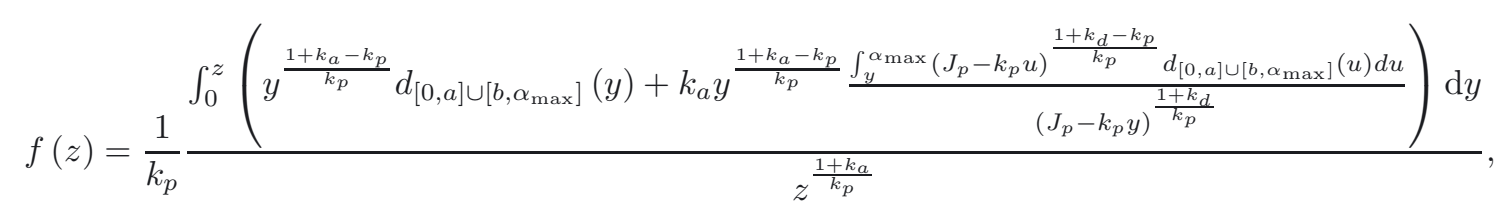

for all $z \in\left(0, \alpha_{\max }\right)$. One notices that

$$
\lim _{z \rightarrow 0+} f(z)=\frac{k_{a}}{\left(1+k_{a}\right) J_{p}^{\frac{1+k_{d}}{k_{p}}}} \int_{a}^{b}\left(J_{p}-k_{p} u\right)^{\frac{1+k_{d}-k_{p}}{k_{p}}} d_{[0, a] \cup\left[b, \alpha_{\max }\right]}(u) \mathrm{d} u>0 .
$$

Therefore, $f(z)>\delta$, for some positive constant $\delta$. In particular, the inequality (4.5) written for $z=x$ gives

$$
\varphi_{n}(x) \leq \frac{k_{a}+k_{d}+1}{k_{a} k_{d}} n^{-1}+a_{n}-\frac{\left(1+k_{a}\right)\left(1+k_{d}\right)}{k_{a} k_{d}} \delta .
$$

We return to (4.5) to obtain

$$
\varphi_{n}(z) \leq \frac{k_{a}+k_{d}+1}{k_{a} k_{d}} n^{-1}+a_{n}-\frac{\left(1+k_{a}\right)\left(1+k_{d}\right)}{k_{a} k_{d}} \delta,
$$

for all $z \in\left(0, \alpha_{\max }\right)$. Finally, taking the supremum over $z \in\left(0, \alpha_{\max }\right)$, yields

$$
0<\delta \leq \frac{k_{a}+k_{d}+1}{\left(1+k_{a}\right)\left(1+k_{d}\right)} n^{-1} .
$$

The last inequality fails to hold for large enough $n$. The assertion of our proposition follows.

Remark 4.4. The reachability result is also true when starting from a generic point $(1, x)$ replacing $(0, x)$.

We now illustrate (Fig. 1) the viability result in Proposition 4.1 and the reachability properties given by Proposition 4.3. We use the classical description of the PDMP associated with Cook's model. The invariant set is represented in green $\left(\left[0, \alpha_{\max }\right]\right)$ and we simulate a trajectory starting from a randomly chosen initial value for the protein. The time horizon is chosen very small (100) and the trajectory is represented in red. The reachable set is given by randomly generated $a, b \in\left(0, \alpha_{\max }\right)$ and is represented by the blue border lines. Whenever the sample remains in the target set for two consecutive time steps, the trajectory is represented in blue (figure in color available online at www.esaim-cocv.org).

\subsection{Bacteriophage $\lambda$}

We consider the model introduced in [17] to describe the regulation of gene expression. The model is derived from the promoter region of bacteriophage $\lambda$. The simplification proposed by the authors of [17] consists in considering a mutant system in which only two operator sites (known as OR2 and OR3) are present. The gene cI expresses repressor (CI), which dimerizes and binds to the DNA as a transcription factor in one of the two available sites. The site OR2 leads to enhanced transcription, while OR3 represses transcription. Using the notations in [17], we let $X$ stand for the repressor, $X_{2}$ for the dimer, $D$ for the DNA promoter site, $D X_{2}$ 

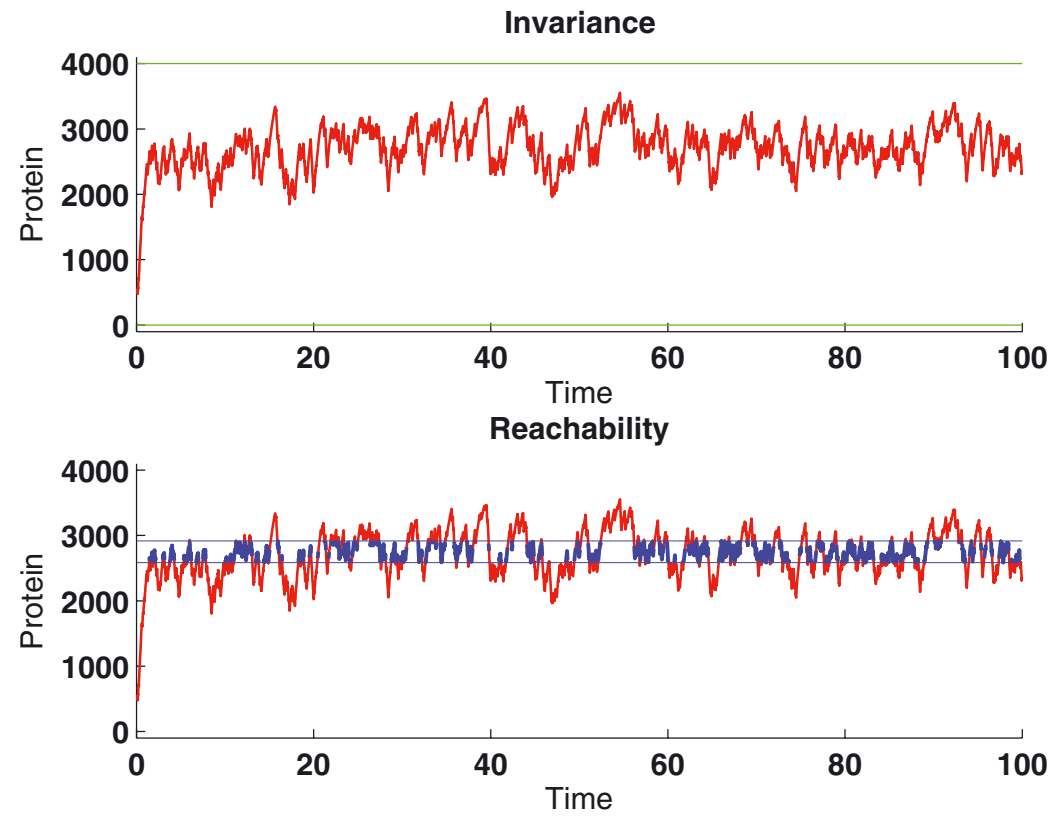

FiguRE 1. Invariance and reachability properties for Cook's model.

for the binding to the OR2 site, $D X_{2}^{*}$ for the binding to the OR3 site and $D X_{2} X_{2}$ for the binding to both sites. We also denote by $P$ the RNA polymerase concentration and by $n$ the number of proteins per mRNA transcript. The dimerization, binding, transcription and degradation reactions are summarized by

$$
\begin{cases}2 X & \stackrel{K_{1}}{\rightleftarrows} X_{2}, \\ D+X_{2} & \stackrel{K_{2}}{\rightleftarrows} D X_{2}, \\ D+X_{2} & \stackrel{K_{3}}{\rightleftarrows} D X_{2}^{*}, \\ D X_{2}+X_{2} & \stackrel{K_{4}}{\rightleftarrows} D X_{2} X . \\ D X_{2}+P & \stackrel{K_{t}}{\longrightarrow} D X_{2}+P+n X \\ X & \stackrel{K_{d}}{\longrightarrow} .\end{cases}
$$

To this biological system we associate a piecewise deterministic process on the state space $E=\left\{\nu \in\{0,1\}^{4}\right.$ : $\left.\sum_{i=1}^{4} \nu_{i}=1\right\} \times \mathbb{R}^{2}$. The characteristic is given by

$$
\begin{aligned}
f_{\nu}\left(x_{1}, x_{2}\right)= & f\left(x_{1}, x_{2}\right)=\left(-2 k_{1} x_{1}{ }^{2}-k_{d} x_{1}+2 k_{-1} x_{2}, k_{1} x_{1}^{2}-k_{-1} x_{2}\right), \\
f(\nu, x)= & \left(0,0,0,0, f_{\nu}(x)\right) \\
\lambda(\nu, x)= & k_{2} x_{2} \chi\left(x_{2}\right) \nu_{1}+k_{3} x_{2} \chi\left(x_{2}\right) \nu_{1}+k_{4} x_{2} \chi\left(x_{2}\right) \nu_{2}+k_{t} \nu_{2}+k_{-2} \nu_{2}+k_{-3} \nu_{3}+k_{-4} \nu_{4}, \\
\lambda(\nu, x) Q((\lambda, x) ; \mathrm{d} z)= & k_{2} x_{2} \chi\left(x_{2}\right) \nu_{1} \delta_{\left(x_{1}, x_{2}-1, \nu_{1}-1, \nu_{2}+1, \nu_{3}, \nu_{4}\right)}(\mathrm{d} z) \\
& +k_{3} x_{2} \chi\left(x_{2}\right) \nu_{1} \delta_{\left(x_{1}, x_{2}-1, \nu_{1}-1, \nu_{2}, \nu_{3}+1, \nu_{4}\right)}(\mathrm{d} z) \\
& +k_{4} x_{2} \chi\left(x_{2}\right) \nu_{2} \delta_{\left(x_{1}, x_{2}-1, \nu_{1}, \nu_{2}-1, \nu_{3}, \nu_{4}+1\right)}(\mathrm{d} z) \\
& +k_{t} \nu_{2} \delta_{\left(x_{1}+n, x_{2}, \nu_{1}, \nu_{2}, \nu_{3}, \nu_{4}\right)}(\mathrm{d} z)+k_{-2} \nu_{2} \delta_{\left(x_{1}, x_{2}+1, \nu_{1}+1, \nu_{2}-1, \nu_{3}, \nu_{4}\right)}(\mathrm{d} z) \\
& +k_{-3} \nu_{3} \delta_{\left(x_{1}, x_{2}+1, \nu_{1}+1, \nu_{2}, \nu_{3}-1, \nu_{4}\right)}(\mathrm{d} z)+k_{-4} \nu_{4} \delta_{\left(x_{1}, x_{2}+1, \nu_{1}, \nu_{2}+1, \nu_{3}, \nu_{4}-1\right)}(\mathrm{d} z),
\end{aligned}
$$


for every $(\nu, x) \in E$. The function $\chi$ is a smooth function such that $0 \leq \chi \leq 1, \chi(y)=0$ for $y<1$ and $\chi(y)=1$ for $y \geq 1+$ err. We consider the stability question for this system. Obviously, whenever a point $\left(\nu^{0}, x^{0}\right)$ is candidate to stability, one should at least expect that this point should be stable with respect to the deterministic evolution. One easily notices that the unique equilibrium point for the ordinary equation driven by the vector field $f_{\nu}$ must satisfy $x^{0}=(0,0)$. Therefore, any candidate for stability with respect to the piecewise deterministic evolution associated to the lambda phage should be of this form.

We shall prove that any small enough region surrounding $(1,0,0,0,0,0)$ is invariant with respect to the PDMP. We emphasize that similar arguments can be used to infer that no other point has similar stability properties. However, different invariant set may exist. Thus, bistability of bacteriophage $\lambda$ should be understood as: a stable state $(0,0,1,0,0,0)$ and some stability (invariance) region.

The main result of the subsection is:

Proposition 4.5. For every $\frac{k_{d}^{2}}{4 k_{1} k_{-1}} \wedge 1>\varepsilon>0$, the set

$$
K_{\varepsilon}=\{(1,0,0,0)\} \times\left[0, \frac{2 k_{-1}}{k_{d}} \varepsilon\right] \times[0, \varepsilon]
$$

is invariant with respect to the PDMP associated to the bacteriophage $\lambda$ model.

Proof. We notice that, for every $\left(x_{1}, x_{2}\right) \in\left[0, \frac{2 k_{-1}}{k_{d}} \varepsilon\right] \times[0, \varepsilon]$, one has

$$
\lambda\left(1,0,0,0, x_{1}, x_{2}\right)=0 .
$$

The following table gives, for all the possible values of $\left(x_{1}, x_{2}\right)$, the explicit form of the normal cone to $\widetilde{K}_{\varepsilon}=$ $\left[0, \frac{2 k_{-1}}{k_{d}} \varepsilon\right] \times[0, \varepsilon]$ at $\left(x_{1}, x_{2}\right)$ and the expression of $\left\langle p, f\left(x_{1}, x_{2}\right)\right\rangle$ for every $p=\left(p_{1}, p_{2}\right) \in \mathbf{N}_{\widetilde{K}_{\varepsilon}}\left(x_{1}, x_{2}\right)$.

\begin{tabular}{|l|l|l|l|}
\hline$x_{1}$ & $x_{2}$ & $\mathbf{N}_{\tilde{K}_{\varepsilon}}\left(x_{1}, x_{2}\right)$ & $\left\langle p, f\left(x_{1}, x_{2}\right)\right\rangle$ \\
\hline 0 & 0 & $\left(\mathbb{R}_{-}\right)^{2}$ & 0 \\
\hline 0 & $(0, \varepsilon)$ & $\mathbb{R}_{-} \times\{0\}$ & $2 p_{1} k_{-1} x_{2}$ \\
\hline 0 & $\varepsilon$ & $\mathbb{R}_{-} \times \mathbb{R}_{+}$ & $\left(2 p_{1}-p_{2}\right) k_{-1} \varepsilon$ \\
\hline$\left(0, \frac{2 k_{-1}}{k_{d}} \varepsilon\right)$ & 0 & $\{0\} \times \mathbb{R}_{-}$ & $p_{2} k_{1} x_{1}^{2}$ \\
\hline$\left(0, \frac{2 k_{-1}}{k_{d}} \varepsilon\right)$ & $(0, \varepsilon)$ & $\{0\} \times\{0\}$ & 0 \\
\hline$\left(0, \frac{2 k_{-1}}{k_{d}} \varepsilon\right)$ & $\varepsilon$ & $\{0\} \times \mathbb{R}_{+}$ & $p_{2}\left(k_{1} x_{1}^{2}-k_{-1} \varepsilon\right)$ \\
\hline$\frac{2 k_{-1}}{k_{d}} \varepsilon$ & 0 & $\mathbb{R}_{+} \times \mathbb{R}_{-}$ & $-p_{1}\left(\frac{8 k_{1} k_{-1}^{2}}{k_{d}^{2}} \varepsilon^{2}+2 k_{-1} \varepsilon\right)+p_{2} \frac{4 k_{1} k_{-1}^{2}}{k_{d}^{2}} \varepsilon^{2}$ \\
\hline$\frac{2 k_{-1}}{k_{d}} \varepsilon$ & $(0, \varepsilon)$ & $\mathbb{R}_{+} \times\{0\}$ & $-p_{1}\left(\frac{8 k_{1} k_{-1}^{2}}{k_{d}^{2}} \varepsilon^{2}+2 k_{-1} \varepsilon-2 k_{-1} x_{2}\right)$ \\
\hline$\frac{2 k_{-1}}{k_{d}} \varepsilon$ & $\varepsilon$ & $\mathbb{R}_{+} \times \mathbb{R}_{+}$ & $-p_{1} \frac{8 k_{1} k_{-1}^{2}}{k_{d}^{2}} \varepsilon^{2}+p_{2} k_{-1} \varepsilon\left(\frac{4 k_{1} k_{-1}}{k_{d}^{2}} \varepsilon-1\right)$ \\
\hline
\end{tabular}

The last column allows to conclude that $\left\langle p, f\left(x_{1}, x_{2}\right)\right\rangle \leq 0$, for all $\left(x_{1}, x_{2}\right) \in\left[0, \frac{2 k_{-1}}{k_{d}} \varepsilon\right] \times[0, \varepsilon]$ and all $p \in \mathbf{N}_{K_{\varepsilon}}\left(x_{1}, x_{2}\right)$. The conclusion follows from Theorem 2.8.

We illustrate the invariance result from the previous proposition in Figure 2. The reader is invited to notice that, in the setting of the previous proposition, the trajectory should be purely deterministic $\left(\lambda\left(1,0,0,0, x_{1}, x_{2}\right)=\right.$ 0 , for every $\left.\left(x_{1}, x_{2}\right) \in\left[0, \frac{2 k_{-1}}{k_{d}} \varepsilon\right] \times[0, \varepsilon]\right)$. We randomly simulate the parameter $\varepsilon<\frac{k_{d}^{2}}{4 k_{1} k_{-1}} \wedge 1$ and a starting point $\left(x_{1}, x_{2}\right) \in\left[0, \frac{2 k_{-1}}{k_{d}} \varepsilon\right] \times[0, \varepsilon]$. The simulated trajectory is represented in red and the bounds $\frac{2 k_{-1}}{k_{d}} \varepsilon$ and $\varepsilon$ are given in green (figure in color available online at www.esaim-cocv.org). 
Invariance for Bacteriophage $\lambda$
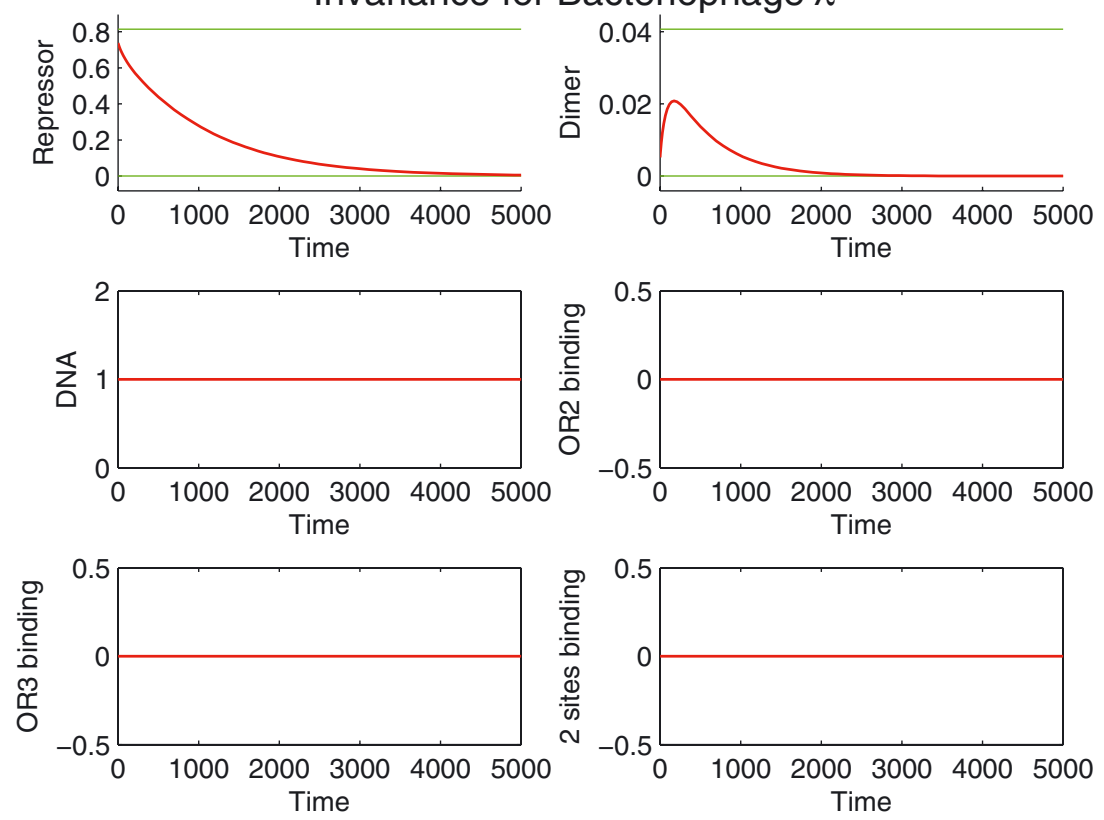

Figure 2. Invariance properties for Bacteriophage $\lambda$.

\section{Appendix}

\subsection{A1}

We begin by sketching the proof of the comparison principle.

Proof of Proposition 2.5. Let us suppose that, for some positive constant $\theta>0$,

$$
\sup _{x \in \mathbb{R}^{N}}(W(x)-V(x))=\theta>0 .
$$

For every $\delta>0$, we define

$$
\theta_{\delta}=\sup _{x \in \mathbb{R}^{N}}\left(W(x)-V(x)-\delta|x|^{2}\right) .
$$

It is obvious that $\left(\theta_{\delta}\right)$ is decreasing and $\lim _{\delta \rightarrow 0} \theta_{\delta}=\theta$. For every $\varepsilon>0$, we introduce

$$
\Phi_{\varepsilon, \delta}(x, y)=W(x)-V(y)-\left|\frac{x-y}{\varepsilon}\right|^{2}-\delta|x|^{2},
$$

for all $x, y \in \mathbb{R}^{N}$. We recall that $W$ and $V$ are bounded. Then, using the u.s.c. of $\Phi_{\varepsilon, \delta}$, we get the existence of some global maximum point $\left(x_{\varepsilon, \delta}, y_{\varepsilon, \delta}\right) \in \mathbb{R}^{2 N}$ of $\Phi_{\varepsilon, \delta}$. Standard arguments yield the existence of some $x_{\delta} \in \mathbb{R}^{N}$ such that $\theta_{\delta}=W\left(x_{\delta}\right)-V\left(x_{\delta}\right)-\delta\left|x_{\delta}\right|^{2}$, and

$$
\left\{\begin{array}{l}
\text { (i) } \lim _{\varepsilon \rightarrow 0}\left|\frac{x_{\varepsilon, \delta}-y_{\varepsilon, \delta}}{\varepsilon}\right|^{2}=0, \text { (ii) } \lim _{\varepsilon \rightarrow 0} x_{\varepsilon, \delta}=\lim _{\varepsilon \rightarrow 0} y_{\varepsilon, \delta}=x_{\delta}, \\
\text { (iii) } \lim _{\varepsilon \rightarrow 0} W\left(x_{\varepsilon, \delta}\right)=W\left(x_{\delta}\right) \text {, (iv) } \lim _{\varepsilon \rightarrow 0} V\left(y_{\varepsilon, \delta}\right)=V\left(x_{\delta}\right) .
\end{array}\right.
$$

We also obtain

$$
\lim _{\delta \rightarrow 0} \delta\left|x_{\delta}\right|^{2}=0 .
$$


We recall that $W$ is a viscosity subsolution and consider the test function

$$
\varphi_{\varepsilon, \delta}(x)=V\left(y_{\varepsilon, \delta}\right)+\left|\frac{x-y_{\varepsilon, \delta}}{\varepsilon}\right|^{2}+\delta|x|^{2},
$$

for all $x \in \mathbb{R}^{N}$. We get

$$
\begin{aligned}
0 \geq & W\left(x_{\varepsilon, \delta}\right)-d_{K}\left(x_{\varepsilon, \delta}\right) \wedge 1+\sup _{u \in U}\left\{-\frac{2}{\varepsilon^{2}}\left\langle f\left(x_{\varepsilon, \delta}, u\right), x_{\varepsilon, \delta}-y_{\varepsilon, \delta}\right\rangle\right. \\
& \left.-2 \delta\left\langle f\left(x_{\varepsilon, \delta}, u\right), x_{\varepsilon, \delta}\right\rangle-\lambda\left(x_{\varepsilon, \delta}, u\right) \int_{\mathbb{R}^{N}}\left(W(z)-W\left(x_{\varepsilon, \delta}\right)\right) Q\left(x_{\varepsilon, \delta}, u, \mathrm{~d} z\right)\right\} . \\
\geq & W\left(x_{\varepsilon, \delta}\right)-d_{K}\left(x_{\varepsilon, \delta}\right) \wedge 1-C \delta\left|x_{\varepsilon, \delta}\right|+\sup _{u \in U}\left\{-\frac{2}{\varepsilon^{2}}\left\langle f\left(x_{\varepsilon, \delta}, u\right), x_{\varepsilon, \delta}-y_{\varepsilon, \delta}\right\rangle\right. \\
& \left.-\lambda\left(x_{\varepsilon, \delta}, u\right) \int_{\mathbb{R}^{N}}\left(W(z)-W\left(x_{\varepsilon, \delta}\right)\right) Q\left(x_{\varepsilon, \delta}, u, \mathrm{~d} z\right)\right\},
\end{aligned}
$$

where $C$ is a generic real constant that may change from one line to another. Standard estimates yield

$$
\begin{aligned}
0 \geq & W\left(x_{\varepsilon, \delta}\right)-d_{K}\left(x_{\varepsilon, \delta}\right) \wedge 1-C\left(\delta\left|x_{\varepsilon, \delta}\right|+\left|W\left(x_{\varepsilon, \delta}\right)-W\left(x_{\delta}\right)\right|+\left|x_{\varepsilon, \delta}-x_{\delta}\right|\right) \\
& +\sup _{u \in U}\left\{-\frac{2}{\varepsilon^{2}}\left\langle f\left(x_{\varepsilon, \delta}, u\right), x_{\varepsilon, \delta}-y_{\varepsilon, \delta}\right\rangle-\lambda\left(x_{\delta}, u\right) \int_{\mathbb{R}^{N}}\left(W(z)-W\left(x_{\delta}\right)\right) Q\left(x_{\varepsilon, \delta}, u, \mathrm{~d} z\right)\right\} .
\end{aligned}
$$

In a similar way, one has

$$
\begin{aligned}
0 \leq & V\left(y_{\varepsilon, \delta}\right)-d_{K}\left(y_{\varepsilon, \delta}\right) \wedge 1+C\left(\left|V\left(y_{\varepsilon, \delta}\right)-V\left(x_{\delta}\right)\right|+\left|y_{\varepsilon, \delta}-x_{\delta}\right|\right) \\
& +\sup _{u \in U}\left\{-\frac{2}{\varepsilon^{2}}\left\langle f\left(y_{\varepsilon, \delta}, u\right), x_{\varepsilon, \delta}-y_{\varepsilon, \delta}\right\rangle-\lambda\left(x_{\delta}, u\right) \int_{\mathbb{R}^{N}}\left(V(z)-V\left(x_{\delta}\right)\right) Q\left(y_{\varepsilon, \delta}, u, \mathrm{~d} z\right)\right\} .
\end{aligned}
$$

Combining (5.5) and (5.6), we get

$$
\begin{aligned}
0 \leq & V\left(y_{\varepsilon, \delta}\right)-W\left(x_{\varepsilon, \delta}\right)+C\left(\left|\frac{x_{\varepsilon, \delta}-y_{\varepsilon, \delta}}{\varepsilon}\right|^{2}+\left|x_{\varepsilon, \delta}-x_{\delta}\right|+\left|y_{\varepsilon, \delta}-x_{\delta}\right|\right) \\
& +C\left(\delta\left|x_{\varepsilon, \delta}\right|+\left|V\left(y_{\varepsilon, \delta}\right)-V\left(x_{\delta}\right)\right|+\left|W\left(x_{\varepsilon, \delta}\right)-W\left(x_{\delta}\right)\right|\right) \\
& +\sup _{u \in U}\left\{\lambda\left(x_{\delta}, u\right)\left(\int_{\mathbb{R}^{N}}\left(W(z)-W\left(x_{\delta}\right)\right) Q\left(x_{\varepsilon, \delta}, u, \mathrm{~d} z\right)-\int_{\mathbb{R}^{N}}\left(V(z)-V\left(x_{\delta}\right)\right) Q\left(y_{\varepsilon, \delta}, u, \mathrm{~d} z\right)\right)\right\} .
\end{aligned}
$$

On the other hand, we notice that

$$
\begin{aligned}
& \int_{\mathbb{R}^{N}}\left(W(z)-W\left(x_{\delta}\right)\right) Q\left(x_{\varepsilon, \delta}, u, \mathrm{~d} z\right)-\int_{\mathbb{R}^{N}}\left(V(z)-V\left(x_{\delta}\right)\right) Q\left(y_{\varepsilon, \delta}, u, \mathrm{~d} z\right) \\
& \leq \int_{\mathbb{R}^{N}}\left(W(z)-V(z)-W\left(x_{\delta}\right)+V\left(x_{\delta}\right)\right) Q\left(x_{\varepsilon, \delta}, u, \mathrm{~d} z\right) \\
& \quad+\int_{\mathbb{R}^{N}} V(z)\left(Q\left(x_{\varepsilon, \delta}, u, \mathrm{~d} z\right)-Q\left(y_{\varepsilon, \delta}, u, \mathrm{~d} z\right)\right) .
\end{aligned}
$$


Thus, whenever $V$ is continuous,

$$
\begin{aligned}
& \int_{\mathbb{R}^{N}}\left(W(z)-W\left(x_{\delta}\right)\right) Q\left(x_{\varepsilon, \delta}, u, \mathrm{~d} z\right)-\int_{\mathbb{R}^{N}}\left(V(z)-V\left(x_{\delta}\right)\right) Q\left(y_{\varepsilon, \delta}, u, \mathrm{~d} z\right) \\
& \leq \int_{\mathbb{R}^{N}}\left(\delta|z|^{2} \wedge C\right) Q\left(x_{\delta}, u, \mathrm{~d} z\right)+\eta_{\left(\delta|\cdot|^{2} \wedge C\right)}\left(\left|x_{\varepsilon, \delta}-x_{\delta}\right|\right)+\eta_{V}\left(\left|x_{\varepsilon, \delta}-y_{\varepsilon, \delta}\right|\right),
\end{aligned}
$$

where $\eta_{\delta}$ and $\eta_{V}$ are given by Assumption (A3) and are independent of $u \in U$. Similar estimates hold true if $W$ is continuous. We substitute (5.8) in (5.7) and take limsup as $\varepsilon \rightarrow 0$ in (5.7) to obtain

$$
\begin{aligned}
0 & \leq-\theta_{\delta}+\delta\left|x_{\delta}\right|+C \sup _{u \in U} \int_{\mathbb{R}^{N}}\left(\delta|z|^{2} \wedge 1\right) Q\left(x_{\delta}, u, \mathrm{~d} z\right) \\
& \leq-\theta_{\delta}+\delta\left|x_{\delta}\right|+C \sup _{u \in U}\left\{\int_{\bar{B}\left(x_{\delta}, \delta^{-\frac{1}{4}}\right)} \delta|z|^{2} Q\left(x_{\delta}, u, \mathrm{~d} z\right)+Q\left(x_{\delta}, u, \mathbb{R}^{N} \backslash \bar{B}\left(x_{\delta}, \delta^{-\frac{1}{4}}\right)\right)\right\} \\
& \leq-\theta_{\delta}+C\left(\delta\left|x_{\delta}\right|^{2}+\delta\left|x_{\delta}\right|+\delta^{\frac{1}{2}}\right)+\sup _{(x, u) \in \mathbb{R}^{N} \times U} Q\left(x, u, \mathbb{R}^{N} \backslash \bar{B}\left(x, \delta^{-\frac{1}{4}}\right)\right) .
\end{aligned}
$$

We allow $\delta \rightarrow 0$ in the last inequality and recall that (A5) holds true to have

$$
0 \leq-\theta
$$

This comes in contradiction with (5.1). The proof is now complete.

\subsection{A2}

The proof of Theorem 3.7 relies on the fact that the functions $V^{\varepsilon}$ defined by (3.20) are viscosity subsolutions of the Hamilton-Jacobi integro-differential equation (3.5). The proof adapts the arguments used in Barles and Jakobsen [9], Lemma 2.7. Following the proof of this lemma, we introduce, for every $h>0, u^{2} \in \mathbb{R}^{N}$, $Q_{h}^{u^{2}}=u^{2}+\left[-\frac{h}{2}, \frac{h}{2}\right)^{N}, \rho_{\varepsilon}^{h, u^{2}}=\int_{Q_{h}^{u^{2}}} \rho_{\varepsilon}(y) \mathrm{d} y$, and $I_{h}(x)=\sum_{u^{2} \in h \mathbb{Z}^{N}} \rho_{\varepsilon}^{h, u^{2}} v^{\varepsilon}\left(x-u^{2}\right)$. Thus, $I_{h}$ is a convex combination of bounded, uniformly continuous viscosity subsolutions of (3.5). Moreover, by classical results, the discretization $I_{h}$ converges uniformly to $V^{\varepsilon}$. To conclude, we show that viscosity subsolutions are preserved by convex combination and uniform convergence.

Proposition 5.1. Given two bounded, uniformly continuous viscosity subsolutions $v_{1}$ and $v_{2}$ of equation (3.5) and two nonnegative real constants $\lambda_{1}, \lambda_{2} \in \mathbb{R}_{+}$such that $\lambda_{1}+\lambda_{2}=1$, the convex combination $\lambda_{1} v_{1}+\lambda_{2} v_{2}$ is still a viscosity subsolution of (3.5).

Proof. The assertion is trivial when either $\lambda_{1}=0$ or $\lambda_{2}=0$. If $\lambda_{1} \lambda_{2} \neq 0$, we let $\bar{x} \in \mathbb{R}^{N}$ and $\varphi \in C_{b}^{1}\left(\mathcal{N}_{\bar{x}}\right)$ be a test function such that

$$
\lambda_{1} v_{1}(\bar{x})+\lambda_{2} v_{2}(\bar{x})-\varphi(\bar{x}) \geq \lambda_{1} v_{1}(y)+\lambda_{2} v_{2}(y)-\varphi(y)
$$

for all $y \in \mathbb{R}^{N}$. We may assume, without loss of generality that $\varphi \in C_{b}\left(\mathbb{R}^{N}\right)$. Indeed, whenever $\varphi$ does not satisfy this assumption, one can replace it with some $\varphi^{0}$ defined as follows: first, notice that there exists some $r>0$ such that $B(\bar{x}, 2 r) \subset \mathcal{N}_{\bar{x}}$. We define

$$
\begin{aligned}
\varphi^{0}(y)= & \left(\varphi(y)+\lambda_{1} v_{1}(\bar{x})+\lambda_{2} v_{2}(\bar{x})-\varphi(\bar{x})\right) \chi(y) \\
& +\left(\lambda_{1} v_{1}(y)+\lambda_{2} v_{2}(y)\right)(1-\chi(y))
\end{aligned}
$$


for all $y \in \mathbb{R}^{N}$, where $\chi$ is a smooth function such that $0 \leq \chi \leq 1, \chi(y)=1$, if $y \in B(\bar{x}, r)$ and $\chi(y)=0$, if $y \in \mathbb{R}^{N} \backslash B(\bar{x}, 2 r)$. Then (5.9) holds true with $\varphi^{0}$ instead of $\varphi$. The new function $\varphi^{0}$ also satisfies

$$
\nabla \varphi^{0}(\bar{x})=\nabla \varphi(\bar{x})
$$

We introduce, for every $\varepsilon>0$

$$
\Phi_{\varepsilon}(x, y)=\lambda_{1} v_{1}(x)+\lambda_{2} v_{2}(y)-\lambda_{1} \varphi(x)-\lambda_{2} \varphi(y)-\frac{1}{\varepsilon^{2}}|x-y|^{2}-|x-\bar{x}|^{2},
$$

for all $x, y \in \mathbb{R}^{N}$. We recall that the functions $v_{1}, v_{2}$ and $\varphi$ are bounded and continuous. This yields the existence of a global maximum $\left(x_{\varepsilon}, y_{\varepsilon}\right)$ of $\Phi_{\varepsilon}$. Moreover, by standard arguments,

$$
\lim _{\varepsilon \rightarrow 0} x_{\varepsilon}=\lim _{\varepsilon \rightarrow 0} y_{\varepsilon}=\bar{x}, \lim _{\varepsilon \rightarrow 0}\left|\frac{x_{\varepsilon}-y_{\varepsilon}}{\varepsilon}\right|^{2}=0 .
$$

We consider the test function $\psi$ given by

$$
\psi(x)=-\lambda_{2} \lambda_{1}^{-1} v_{2}\left(y_{\varepsilon}\right)+\varphi(x)+\lambda_{2} \lambda_{1}^{-1} \varphi\left(y_{\varepsilon}\right)+\frac{\lambda_{1}^{-1}}{\varepsilon^{2}}\left|x-y_{\varepsilon}\right|^{2}+\lambda_{1}^{-1}|x-\bar{x}|^{2},
$$

for all $x \in \mathbb{R}^{N}$. We recall that the function $v_{1}$ is a viscosity subsolution for (3.5). Then,

$$
v_{1}\left(x_{\varepsilon}\right)+d_{\mathcal{O}^{c}}\left(x_{\varepsilon}\right) \wedge 1+H\left(x_{\varepsilon}, \nabla \varphi\left(x_{\varepsilon}\right)+\frac{2 \lambda_{1}^{-1}}{\varepsilon^{2}}\left(x_{\varepsilon}-y_{\varepsilon}\right)+2 \lambda_{1}^{-1}\left(x_{\varepsilon}-\bar{x}\right), v_{1}\right) \leq 0 .
$$

Standard estimates yield

$$
\begin{aligned}
0 \geq & v_{1}(\bar{x})+d_{\mathcal{O}^{c}}(\bar{x}) \wedge 1+\sup _{u \in U}\left\{-\langle f(\bar{x}, u), \nabla \varphi(\bar{x})\rangle-\frac{2 \lambda_{1}^{-1}}{\varepsilon^{2}}\left\langle x_{\varepsilon}-y_{\varepsilon}, f\left(x_{\varepsilon}, u\right)\right\rangle\right. \\
& \left.-\lambda(\bar{x}, u) \int_{\mathbb{R}^{N}}\left(v_{1}(z)-v_{1}(\bar{x})\right) Q(\bar{x}, u, \mathrm{~d} z)\right\} \\
& -C\left(\left|x_{\varepsilon}-\bar{x}\right|+\left|v_{1}\left(x_{\varepsilon}\right)-v_{1}(\bar{x})\right|+\left|\nabla \varphi\left(x_{\varepsilon}\right)-\nabla \varphi(\bar{x})\right|+\eta_{v_{1}}\left(\left|x_{\varepsilon}-\bar{x}\right|\right)\right) .
\end{aligned}
$$

In a similar way, we get

$$
\begin{aligned}
0 \geq & v_{2}(\bar{x})+d_{\mathcal{O}^{c}}(\bar{x}) \wedge 1+\sup _{u \in U}\left\{-\langle f(\bar{x}, u), \nabla \varphi(\bar{x})\rangle+\frac{2 \lambda_{2}^{-1}}{\varepsilon^{2}}\left\langle x_{\varepsilon}-y_{\varepsilon}, f\left(y_{\varepsilon}, u\right)\right\rangle\right. \\
& \left.-\lambda(\bar{x}, u) \int_{\mathbb{R}^{N}}\left(v_{2}(z)-v_{2}(\bar{x})\right) Q(\bar{x}, u, \mathrm{~d} z)\right\} \\
& -C\left(\left|y_{\varepsilon}-\bar{x}\right|+\left|v_{2}\left(y_{\varepsilon}\right)-v_{2}(\bar{x})\right|+\left|\nabla \varphi\left(y_{\varepsilon}\right)-\nabla \varphi(\bar{x})\right|+\eta_{v_{2}}\left(\left|y_{\varepsilon}-\bar{x}\right|\right)\right) .
\end{aligned}
$$

Finally, using (5.11), (5.12) and (5.10), and passing to the limit as $\varepsilon \rightarrow 0$, yields

$$
\left(\lambda_{1} v_{1}+\lambda_{2} v_{2}\right)(\bar{x})+d_{\mathcal{O}^{c}}(\bar{x}) \wedge 1+H\left(\bar{x}, \nabla \varphi(\bar{x}), \lambda_{1} v_{1}+\lambda_{2} v_{2}\right) \leq 0 .
$$

These arguments allow to obtain, by recurrence, that any convex combination of continuous, bounded viscosity subsolutions is still a subsolution for (3.5).

Proposition 5.2 (stability). Let $\left(v_{n}\right)_{n}$ be a sequence of continuous, uniformly bounded viscosity subsolutions of (3.5). Moreover, we suppose that $v_{n}$ converges uniformly on compact sets to some continuous, bounded function $v$. Then the function $v$ is a viscosity subsolution of (3.5). 
Proof. We let $x \in \mathbb{R}^{N}$ and $\varphi \in C_{b}^{1}\left(\mathcal{N}_{x}\right)$ be a test function such that $v-\varphi$ has a global maximum at $x$. As in the previous proposition, one can assume, without loss of generality, that $\varphi \in C_{b}\left(\mathbb{R}^{N}\right)$. Classical arguments yield the existence of some point $x_{n} \in \mathbb{R}^{N}$ such that

$$
v_{n}\left(x_{n}\right)-\varphi\left(x_{n}\right)-\left|x_{n}-x\right|^{2} \geq v_{n}(y)-\varphi(y)-|y-x|^{2},
$$

for all $y \in \mathbb{R}^{N}$ and

$$
\lim _{n \rightarrow \infty} x_{n}=x .
$$

We assume, without loss of generality, that $\left|x_{n}-x\right| \leq 1$, and $x_{n} \in \mathcal{N}_{x}$, for all $n \geq 1$. Then,

$$
0 \geq v_{n}\left(x_{n}\right)+d_{\mathcal{O}^{c}}\left(x_{n}\right) \wedge 1+\sup _{u \in U}\left\{\begin{array}{c}
-\left\langle f\left(x_{n}, u\right), \nabla \varphi\left(x_{n}\right)+2\left(x_{n}-x\right)\right\rangle \\
-\lambda\left(x_{n}, u\right) \int_{\mathbb{R}^{N}}\left(v_{n}(z)-v_{n}\left(x_{n}\right)\right) Q\left(x_{n}, u, \mathrm{~d} z\right)
\end{array}\right\} .
$$

We have

$$
-\left\langle f\left(x_{n}, u\right), \nabla \varphi\left(x_{n}\right)+2\left(x_{n}-x\right)\right\rangle \geq-\langle f(x, u), \nabla \varphi(x)\rangle-C\left(\left|x_{n}-x\right|+\left|\nabla \varphi\left(x_{n}\right)-\nabla \varphi(x)\right|\right),
$$

where $C>0$ is a generic constant independent of $n \geq 1$ and $u \in U$ which may change from one line to another. We also get

$$
\begin{aligned}
& -\lambda\left(x_{n}, u\right) \int_{\mathbb{R}^{N}}\left(v_{n}(z)-v_{n}\left(x_{n}\right)\right) Q\left(x_{n}, u, \mathrm{~d} z\right) \\
& \geq-\lambda(x, u) \int_{\mathbb{R}^{N}}(v(z)-v(x)) Q(x, u, \mathrm{~d} z)-C\left(\left|x_{n}-x\right|+\left|v_{n}\left(x_{n}\right)-v(x)\right|+\eta_{v}\left(\left|x_{n}-x\right|\right)\right) \\
& \quad-C \sup _{u \in U} \int_{\mathbb{R}^{N}}\left|v_{n}(z)-v(z)\right| Q\left(x_{n}, u, \mathrm{~d} z\right) .
\end{aligned}
$$

Finally, for every $m \geq 1$,

$$
\begin{aligned}
& \sup _{u} \int_{\mathbb{R}^{N}}\left|v_{n}(z)-v(z)\right| Q\left(x_{n}, u, \mathrm{~d} z\right) \\
& \leq \sup _{z \in \bar{B}(0, m+|x|+1)}\left(\left|v_{n}(z)-v(z)\right|\right)+C \sup _{u \in U} Q\left(x_{n}, u, \mathbb{R}^{N} \backslash \bar{B}(0, m+|x|+1)\right) \\
& \leq \sup _{z \in \bar{B}(0, m+|x|+1)}\left(\left|v_{n}(z)-v(z)\right|\right)+C \sup _{u \in U} Q\left(x_{n}, u, \mathbb{R}^{N} \backslash \bar{B}\left(x_{n}, m\right)\right) \\
& \leq \sup _{z \in \bar{B}(0, m+|x|+1)}\left(\left|v_{n}(z)-v(z)\right|\right)+C \sup _{y \in \mathbb{R}^{N}, u \in U} Q\left(y, u, \mathbb{R}^{N} \backslash \bar{B}(y, m)\right) .
\end{aligned}
$$

We substitute (5.14)-(5.16) in (5.13) and allow $n \rightarrow \infty$ to have

$$
\begin{aligned}
0 \geq & v(x)+d_{\mathcal{O}^{c}}(x) \wedge 1+\sup _{u \in U}\left\{-\langle f(x, u), \nabla \varphi(x)\rangle-\lambda(x, u) \int_{\mathbb{R}^{N}}(v(z)-v(x)) Q(x, u, \mathrm{~d} z)\right\} \\
& -C \sup _{y \in \mathbb{R}^{N}, u \in U} Q\left(y, u, \mathbb{R}^{N} \backslash \bar{B}(y, m)\right)
\end{aligned}
$$

for all $m \geq 1$. We conclude using the Assumption (A5). 


\section{REFERENCES}

[1] O. Alvarez and A. Tourin, Viscosity solutions of nonlinear integro-differential equations. Ann. Inst. Henri Poincaré, Anal. non linéaire 13 (1996) 293-317.

[2] J.-P. Aubin, Viability Theory. Birkhäuser (1992).

[3] J.-P. Aubin and G. Da Prato, Stochastic viability and invariance. Ann. Sc. Norm. Pisa 27 (1990) 595-694.

[4] J.-P. Aubin and H. Frankowska, Set Valued Analysis. Birkhäuser (1990).

[5] M. Bardi and I. Capuzzo-Dolcetta, Optimal control and viscosity solutions of Hamilton-Jacobi- Bellman equations. Systems and Control: Foundations and Applications, Birkhäuser (1997).

[6] M. Bardi and P. Goatin, Invariant sets for controlled degenerate diffusions: a viscosity solutions approach, in Stochastic analysis, control, optimization and applications, Systems Control Found. Appl., Birkhäuser, Boston, MA (1999) 191-208.

[7] M. Bardi and R. Jensen, A geometric characterization of viable sets for controlled degenerate diffusions. Set-Valued Anal. 10 (2002) 129-141.

[8] G. Barles and C. Imbert, Second-order elliptic integro-differential equations: Viscosity solutions theory revisited. Ann. Inst. Henri Poincaré, Anal. non linéaire 25 (2008) 567-585.

[9] G. Barles and E.R. Jakobsen, On the convergence rate of approximation schemes for Hamilton-Jacobi-Bellman equations. ESAIM: M2AN 36 (2002) 33-54.

[10] R. Buckdahn, S. Peng, M. Quincampoix and C. Rainer, Existence of stochastic control under state constraints. C. R. Acad. Sci. Paris Sér. I Math. 327 (1998) 17-22.

[11] R. Buckdahn, D. Goreac and M. Quincampoix, Stochastic optimal control and linear programming approach. Appl. Math. Opt. 63 (2011) 257-276.

[12] D.L. Cook, A.N. Gerber and S.J. Tapscott, Modelling stochastic gene expression: Implications for haploinsufficiency. Proc. Natl. Acad. Sci. USA 95 (1998) 15641-15646.

[13] A. Crudu, A. Debussche and O. Radulescu, Hybrid stochastic simplifications for multiscale gene networks. BMC Systems Biology 3 (2009).

[14] M.H.A. Davis, Markov Models and Optimization, Monographs on Statistics and Applied probability 49. Chapman \& Hall (1993).

[15] M. Delbrück, Statistical fluctuations in autocatalytic reactions. J. Chem. Phys. 8 (1940) 120-124.

[16] S. Gautier and L. Thibault, Viability for constrained stochastic differential equations. Differential Integral Equations 6 (1993) $1395-1414$.

[17] J. Hasty, J. Pradines, M. Dolnik and J.J. Collins, Noise-based switches and amplifiers for gene expression. PNAS 97 (2000) 2075-2080.

[18] H.M. Soner, Optimal control with state-space constraint. II. SIAM J. Control Optim. 24 (1986) 1110-1122.

[19] X. Zhu and S. Peng, The viability property of controlled jump diffusion processes. Acta Math. Sinica 24 (2008) 1351-1368. 